Eficiencia del gasto en salud en la OCDE y ALC: un análisis envolvente de datos

Daysi Sanmartín-Durango, María Alejandra Henao-Bedoya, Yair Tadeo Valencia-Estupiñán y Jairo Humberto Restrepo-Zea 
Lecturas de Economía, 91 (julio-diciembre 2019), pp. 41-78

Daysi Sanmartin-Durango, María Alejandra Henao-Bedoya, Yair Tadeo Valencia-Estupiñán y Jairo Humberto Restrepo-Zea

\title{
Eficiencia del gasto en salud en la OCDE y ALC: un análisis envolvente de datos
}

Resumen: Este artículo mide la eficiencia del gasto en salud en sesenta y dos países de América Latina y el Caribe (ALC) y de la Organización para la Cooperación y el Desarrollo Económico (OCDE), a partir de la relación entre el nivel de gasto total (como porcentaje del PIB) y algunos resultados en salud (esperan₹a de vida en años y mortalidad en menores de cinco años por cada mil nacidos vivos). Con este fin, se aplicó el método no paramétrico de análisis envolvente de datos, usando para cada grupo datos de 1995, 2005 y 2014. Los resultados permiten identificar la eficiencia y la posición relativa del conjunto de paises dentro de ambos grupos. Para el año 2014, los países más eficientes de ALC fueron Chile, Cuba, República Dominicana, Venezuela y Jamaica, y de la OCDE fueron Japón, Luxemburgo y Turquía. El promedio de la eficiencia de los paises de ALC fue inferior a la de los países la OCDE (0,938 y 0,974, respectivamente).

Palabras clave: gasto en salud; eficiencia; análisis envolvente de datos; América Latina y el Caribe; Organización para la Cooperación y el Desarrollo Económico.

Clasificación JEL: I19, H21, C14, O54, O57.

\section{Efficiency of health expenditure in the OECD and LAC: a data envelopment analysis}

\begin{abstract}
This paper measures the efficiency of expenditure in health care in 62 countries of Latin America and the Caribbean (LAC) and the Organization for Economic Co-operation and Development (OECD), based on the ratio between the level of total expenditure (as percent of GDP) and some bealth results (life expectancy and mortality rates in children under five years of age per every 1000 children born alive). For this purpose, the non-parametric method data envelopment analysis was applied using data from 1995, 2005 and 2014 for each group. The results allow identifying the relative efficiency and position of the set of countries analysed within both groups of countries. In 2014, the most efficient countries in LAC were Chile, Cuba, Dominican Republic, Venezuela and Jamaica, whilst in the OECD these were Japan, Luxembourg and Turkey. The average efficiency of $L A C$ countries turns out to be below that of the $O E C D(0.938$ and 0.974 , respectively).
\end{abstract}

Keywords: bealth expenditure; efficiency; data envelopment analysis; Latin America and the Caribbean; Organization for Economic Cooperation and Development.

JEL Classification: 119, H21, C14, O54, O57.

Efficacité des dépenses de santé dans les pays de l'OCDE et d'Amérique latine et des Caraïbes : une analyse par enveloppement des données

Résumé: Cet article mesure l'efficacité des dépenses de santé dans soixante-deux pays d'Amérique latine et des Caraïbes (ALC) et de l'Organisation de coopération et de développement économiques (OCDE), sur la base du rapport entre le niveau de dépenses total (en pourcentage du PIB) et certains résultats en matière de santé (espérance de vie en années et mortalité chez. les enfants de moins de cinq ans pour mille naissances vivantes). À cette fin, la méthode non paramétrique d'analyse par enveloppement des données a été appliquée à l'aide des données de 1995, 2005 et 2014. Ces résultats permettent d'identifier l'efficacité et la position relative du groupe de pays au sein des deux groupes. Pour l'année 2014, les pays les plus efficaces en Amérique latine et dans les Caraïbes étaient le Chili, Cuba, la République dominicaine, le Venezuela et la Jamä̈que, et l'OCDE, le Japon, le Luxembourg et la Turquie. L'efficacité moyenne des pays d'Amérique latine et des Caraïbes était inférieure à celle des pays de l'OCDE (0,938 et 0,974, respectivement).

Mots clés: dépense de santé; l'efficacité; analyse par enveloppement des données; Amérique latine et les Caraïbes; Organisation de coopération et de développement économiques.

Classification JEL: 119, H21, C14, O54, O57. 


\title{
Eficiencia del gasto en salud en la OCDE y ALC: un análisis envolvente de datos
}

\author{
Daysi Sanmartín-Durango $\mathbb{(}^{\mathrm{a}}$, Maria Alejandra Henao-Bedoya $\mathbb{(}^{\mathrm{b}}$, Yair \\ Tadeo Valencia-Estupiñán (10) y Jairo Humberto Restrepo-Zea ${ }^{\mathrm{d}}{ }^{\mathrm{d}}$
}

\section{-Introducción. -I. Marco conceptual. -II. Hechos estilizados. -III Metodología y datos. -IV. Discusión de resultados. -Conclusiones. -Referencias. -Anexos.}

doi: 10.17533/udea.le.n91a02

Primera versión recibida el 15 de marzo de 2018; versión final aceptada el 13 de diciembre de 2018

\section{Introducción}

Las sociedades se enfrentan a una demanda de servicios de salud que resulta creciente, en gran medida, debido a los cambios demográficos y epidemiológicos, al aumento de la carga de la enfermedad y al desarrollo tecnológico (Clements et al., 2012). Estos factores suponen una presión, ya que aumentan el gasto en salud, el cual pasó de representar el 8,5\% del PIB en 1995 al 9,9\% en 2014

a Daysi Sanmartín-Durango: estudiante de maestría en Economía, Facultad de Ciencias Económicas, Universidad de Antioquia, Medellín, Colombia. Dirección postal: Calle $67 \mathrm{~N}^{\circ}$ 53-108. Dirección electrónica: daysi.sanmartin@udea.edu.co.

https://orcid.org/0000-0002-8653-4876

b Maria Alejandra Henao-Bedoya: analista de cuantificación y analítica de riesgo, Tuya Compañía de Financiamiento, Medellín, Colombia. Dirección postal: Calle 4sur N 43-109. Dirección electrónica: MHenaoB@tuya.com.co. https://orcid.org/0000-0002-1208-4198

c Yair Tadeo Valencia-Estupiñan: analista de gestión de portafolio de servicios no financieros, Dirección de Estrategia de Cliente Pyme y Gobierno, Grupo Bancolombia, Medellín, Colombia. Dirección postal: Carrera 48 N²8-85. Dirección electrónica: yaivalen@bancolombia.com.co. https://orcid.org/0000-0003-2610-4708

d Jairo Humberto Restrepo-Zea: profesor titular y coordinador del Grupo de Economía de la Salud, Facultad de Ciencias Económicas, Universidad de Antioquia, Medellín, Colombia. Dirección postal: Calle 67 N 53-108. Dirección electrónica: jairo.restrepo@udea.edu.co. https://orcid.org/0000-0002-3449-0373 
Sanmartín-Durango et al.: Eficiencia del gasto en salud en la OCDE y ALC...

Un gasto público del 6\% del PIB destinado a la salud (Xu et al., 2010), acordado por los países miembros de la Organización Panamericana de la Salud (OPS), es una referencia útil en la mayoría de los casos y una condición necesaria, aunque no suficiente, para reducir las inequidades y aumentar la protección financiera en el marco de la Cobertura Universal de Salud (CUS) (OMS \& OPS, 2014). Para el año 2014, el gasto público en salud promedio mundial llegó a un $6 \%$ del PIB, aunque con diferencias entre países. Por ejemplo, para los países de la Organización para la Cooperación y el Desarrollo Económico (OCDE) fue de 7,6\%, y en los países de América Latina y el Caribe (ALC) de 3,7\% (datos Banco Mundial, 2015).

Ahora bien, resulta necesario establecer una relación entre los resultados en salud y el gasto público en el sector. Autores como Lago et al. (2013) y Rivera (2001) constatan que esta relación es positiva; así que, mediante un aumento de los recursos dirigidos a la salud, se logran resultados como la disminución de la mortalidad materno-infantil o el incremento en la esperanza de vida. No obstante, otros estudios también establecen que, si bien el aumento de los recursos es fundamental para mejorar la salud en los países pobres, debe reconocerse que se pueden obtener beneficios importantes mediante el uso de los recursos existentes de manera más eficiente (Alfonso $\&$ St. Aubyn, 2004; Evans, et al., 2000).

Se tienen casos particulares de países que, a pesar de tener un reducido gasto en salud, presentan resultados relativamente favorecedores. Es el caso de Perú, que, a pesar de mantener un bajo gasto en salud, entre 2000 y 2013 ocupó el primer lugar en la reducción de la mortalidad neonatal y el segundo en la reducción de la mortalidad en menores de cinco años, entre 75 países de ingresos medios y bajos (Huicho et al., 2016). De manera general, en el informe de 2010 sobre la salud en el mundo, la Organización Mundial de la Salud (OMS) afirma que entre el $20 \%$ y el $40 \%$ del gasto sanitario se desperdicia por ineficiencia. En este contexto, se propone un conjunto de estrategias que tienen como finalidad lograr el ahorro del gasto que los países destinan a salud y aumentar sus niveles de eficiencia (OMS, 2010).

Lo anterior constituye una de las principales motivaciones de este artículo, el cual tiene como objetivo cuantificar la eficiencia relativa del gasto total en 
salud a partir de la relación entre resultados y el nivel de gasto total en salud de los países de ALC y la OCDE. El artículo se divide en seis secciones, incluida esta introducción. En la sección I, se define el marco teórico relacionado con el contexto de la relación salud y desarrollo, el gasto, los resultados en salud y la eficiencia. En la sección II, se hace un análisis descriptivo de un conjunto de datos que permiten caracterizar la evolución de las principales variables de gasto y los resultados en salud de los países en estudio, considerados como hechos estilizados. La metodología del análisis envolvente de datos $(\text { DEA })^{1}$, la selección de los datos y las variables del modelo se describen en la sección III. A continuación, en la sección IV, se exponen los principales resultados obtenidos. Por último, se presentan las conclusiones de acuerdo con los objetivos y los resultados planteados.

\section{Marco conceptual}

En la medida en que el mundo ha logrado un mayor desarrollo, ha sido posible obtener logros importantes en materia de salud, como aumentar la expectativa de vida, disminuir la mortalidad materna e infantil y, en general, mejorar el bienestar de la población (Grossman, 1999). Sin embargo, otros hechos implícitos del desarrollo se refieren a la transición demográfica, al envejecimiento de la población, a las consecuencias de la contaminación y, en general, a los nuevos retos que enfrentan los sistemas de salud dados los patrones urbanos, de vivienda y alimentación (Jackson \& Ugalde, 1987). Por ello, es importante relacionar el nivel de gasto en salud con sus resultados directos y la eficiencia del sector, pues esos resultados toman verdadera relevancia a la hora de reconocer los determinantes de éxito, los cuales, posteriormente, se verán reflejados en el mejoramiento de las condiciones de vida.

\section{A. El gasto y los resultados en salud}

De acuerdo con Xu, Saksena y Holly (2011), el gasto en salud se define como uno de los elementos más importantes de los sistemas de salud para

1 Sigla del término en inglés Data Envelopment Analysis 
Sanmartín-Durango et al.: Eficiencia del gasto en salud en la OCDE y ALC...

ayudar a los formuladores de política pública a planificar un mejor futuro. Algunos trabajos aseguran de manera más enfática que un aumento en los gastos de salud pública conduciría automáticamente a una mejora en el estado de salud. Con esta hipótesis, Rivera (2001) identifica y cuantifica las relaciones entre los distintos niveles de la salud y los recursos teniendo en cuenta un conjunto de factores económicos y sociobiológicos que influyen en la salud del individuo.

Sin embargo, es importante reconocer que una relación efectiva entre gasto y mejoras en salud debe cumplir condiciones adecuadas que enmarquen la relación de los agentes con el sistema. Por ejemplo, Payne et al. (2007) hacen énfasis en el efecto que tiene un aumento en la esperanza de vida sobre el gasto en salud, y demuestran que si la morbilidad no disminuye, o se mantiene constante, puede representar un factor de presión para los gastos de salud. Además, las diferencias entre países enseñan que no todos los territorios logran los mismos resultados con un aumento del gasto. Dieleman et al. (2016) concluyen que, a pesar de los beneficios sanitarios notables, las tendencias de financiación de la salud y las relaciones sugieren que muchos países de ingresos bajos y medios no alcanzarán los objetivos de nivel de gasto en salud internacional.

Algunas investigaciones empíricas han tratado de plasmar la relación entre el gasto en salud y otras variables macroeconómicas. Lago et al. (2013) analizan información de 193 naciones y, si bien no encuentran evidencias a partir de las cuales se pueda concluir la existencia de causalidad, el principal hallazgo es la relación positiva y estable entre los resultados en salud, el gasto en salud per cápita y el ingreso nacional per cápita. Concluyen que la relación entre el gasto en salud per cápita y el estatus de salud sugiere rendimientos decrecientes a escala del gasto.

Por otro lado, mediante un análisis de panel de datos de 153 países para el periodo 1995-2008, Moreno-Serra y Smith (2015) muestran que la cobertura de salud, especialmente por la vía de mayores niveles de gasto en salud financiado con fondos públicos, se traduce en una menor mortalidad, que tiene un efecto mayor sobre la mortalidad infantil y que, adicionalmente, resulta superior en los países más pobres. 


\section{B. La eficiencia macro en el sector salud}

En teoría económica, la eficiencia es un concepto que describe la relación entre insumos y resultados en la producción de bienes y servicios. Sin embargo, para el contexto en el que se desarrolla este artículo, se aborda la eficiencia macro en el sector salud como el grado en que un sistema de salud efectúa la máxima contribución a las metas sociales definidas -como disminuir la morbilidad y la mortalidad y alargar la vida de las personascon los recursos disponibles (Lam \& Hernández, 2008). Particularmente, será considerada desde la eficiencia técnica, la cual supone conseguir determinados resultados a partir de un mínimo de recursos o, alternativamente, lograr el máximo beneficio posible con unos recursos limitados.

En cuanto a las medidas de eficiencia, Aguilar (2010) realizó una revisión bibliográfica de 36 autores, organizaciones y programas de trabajo sobre distintos enfoques de salud y economía. Encontró que, sin excepción, la bibliografía coincide en la necesidad de estudiar la eficiencia de los servicios sanitarios, por lo que recomienda aplicar el DEA para este tipo de estudios, pues resultó predominante como procedimiento de estimación de los índices de eficiencia relativa.

Dieleman et al. (2016) enfatizan la importancia de obtener ganancias vía eficiencia y priorización del gasto, ya que, si bien se necesita aumentar los recursos, también es fundamental proponer una acción concertada para los mismos. Tanto para las economías con un alto índice de desarrollo como para aquellas con un índice medio, el aumento de la eficiencia de los gastos parece ser la única opción para superar la presión de los gastos asociados con el incremento de la edad (Heller \& Hauner, 2006). Se tiene así el reto de mostrar que no siempre un alto nivel de eficiencia implica un aumento significativo en el gasto en salud; es decir, los datos permitirán dar cuenta de que existen sistemas capaces de garantizar buenos resultados en salud sin necesidad de tener un gasto alto. 
Sanmartín-Durango et al.: Eficiencia del gasto en salud en la OCDE y ALC...

\section{Hechos estilizados}

Se identifican cuatro hechos destacados en relación con las variables de gasto y resultados en salud, los cuales motivan la aplicación de un método más preciso, como el DEA, para definir la eficiencia y que, además, permiten plantear intuiciones preliminares de los resultados que podría arrojar la metodología mencionada. Cada hecho se expresa en términos de una hipótesis que puede desarrollarse en el proceso de la investigación.

\section{A. Aunque el gasto público en los países de ALC es creciente, es mayor en los países de la $O C D E$}

Se observa que entre los países de la OCDE la mayoría tienen un gasto público superior al $50 \%$ del gasto total, con excepción de Chile, México y EE.UU. Además, la tendencia no varió significativamente en el periodo 19952014. En 2014 nueve países tenían un gasto público superior al $80 \%$.

Por su parte, en el mismo periodo, en ALC el gasto público tuvo un comportamiento creciente, con un incremento cercano a 3,8 puntos porcentuales. En 1995, la mayoría de países tenían un gasto público inferior al $50 \%$. Esta tendencia ha venido cambiando y en 2014 solo 8 de 29 países continuaban por debajo del $50 \%$. Se destacan los casos de Cuba, con un $96 \%$ del gasto público en 2014, y Panamá, Costa Rica, Bolivia, Uruguay y Colombia, con una participación del gasto público superior al $70 \%$.

En ese sentido, se observa que la composición público-privada del gasto total en salud difiere entre los grupos de países. Sin embargo, en ambos grupos el común denominador ha sido el incremento de la participación del gasto público dentro del gasto total.

\section{B. Gastar más no siempre implica mejores resultados en salud}

En este punto, se elaboró un ranking de países para cada variable en el año 2014. Según la clasificación correspondiente a la variable gasto en salud, se analizaron cinco países de cada grupo: los dos primeros países, un país ubicado en la mediana y los dos últimos países. 
En el grupo de la OCDE, la Tabla 1 muestra casos como el de Estados Unidos que, si bien presenta el gasto más alto, dados los resultados, no se encuentra bien posicionado y su ascenso es lento. Después están Suecia e Italia que, aunque tienen un gasto relativamente alto, muestran resultados aceptables a ese nivel de gasto. Finalmente, México y Turquía son los países que menos gastan en el grupo e, igualmente, se encuentran en los últimos lugares en cuanto a resultados en salud.

Tabla 1. Ranking de paises miembros de la OCDE

\begin{tabular}{lccccc}
\hline & Estados Unidos & Suecia & Italia & México & Turquía \\
\hline Gasto en salud & $1^{\circ}$ & $2^{\circ}$ & $17^{\circ}$ & $33^{\circ}$ & $34^{\circ}$ \\
Esperanza de vida al nacer & $27^{\circ}$ & $12^{\circ}$ & $4^{\circ}$ & $31^{\circ}$ & $34^{\circ}$ \\
Mortalidad infantil & $30^{\circ}$ & $7^{\circ}$ & $11^{\circ}$ & $33^{\circ}$ & $34^{\circ}$ \\
Mortalidad materna & $30^{\circ}$ & $8^{\circ}$ & $7^{\circ}$ & $34^{\circ}$ & $32^{\circ}$ \\
& & & & & \\
\hline
\end{tabular}

Fuente: elaboración propia a partir de datos del Banco Mundial.

Por su parte, para ALC la Tabla 2 da cuenta de que Cuba es el país que más gasta en salud y, además, se encuentra en las primeras posiciones en cuanto a los resultados. Le sigue Paraguay, que resulta ser el segundo país con mayor porcentaje del gasto, pero tiene resultados bajos en comparación con el nivel de gasto en el que se ubica. Colombia se encuentra en el medio en términos tanto de gasto como de resultados, mientras que Venezuela y República Dominicana están ubicados en los últimos lugares con un gasto bajo, pero obtienen resultados aceptables a ese nivel.

Se puede concluir que un mayor gasto en salud no necesariamente genera mayor eficiencia, debido a que, en ocasiones, la ineficiencia puede proceder de un gasto excesivo. 
Sanmartín-Durango et al.: Eficiencia del gasto en salud en la OCDE y ALC...

Tabla 2. Ranking de paises de ALC

\begin{tabular}{lccccc}
\hline Cuba & Paraguay & Colombia & Venezuela & República & Dominicana \\
\hline Gasto en salud & $1^{\circ}$ & $2^{\circ}$ & $14^{\circ}$ & $26^{\circ}$ & $28^{\circ}$ \\
Esperanza de vida al nacer & $3^{\circ}$ & $19^{\circ}$ & $15^{\circ}$ & $22^{\circ}$ & $16^{\circ}$ \\
Mortalidad infantil & $1^{\circ}$ & $20^{\circ}$ & $13^{\circ}$ & $10^{\circ}$ & $25^{\circ}$ \\
Mortalidad materna & $8^{\circ}$ & $20^{\circ}$ & $13^{\circ}$ & $20^{\circ}$ & $21^{\circ}$ \\
& & & & & \\
\hline
\end{tabular}

Fuente: elaboración propia a partir de datos del Banco Mundial.

\section{El efecto del gasto en los resultados en salud difiere según el nivel de desarrollo de los países}

El gasto en salud presenta una relación positiva con la esperanza de vida (Figura 1), y negativa con la mortalidad infantil (Figura 2) y la mortalidad materna. Además, se encuentra un incremento notable en el gasto en salud para el promedio de los miembros de la OCDE, pero la mejora de los resultados para ALC es mayor si se compara la evolución entre los dos años analizados. Por ejemplo, en el periodo 1995-2014, para ALC el aumento de la esperanza de vida fue de 5 años, la reducción de la tasa de mortalidad infantil fue de 24 niños por cada 1.000 nacidos vivos y la disminución en la tasa de mortalidad materna fue de 50 muertes por cada 100.000 nacidos. En cambio, para el caso de los países de la OCDE, los datos fueron, respectivamente, 4 años de aumento de la esperanza de vida, 16 muertes infantiles y 9 muertes maternas.

El efecto marginal de un aumento porcentual del gasto en salud ( $\%$ PIB) sobre los resultados en salud es diferente entre países según su nivel de desarrollo. Es decir, el impacto es mayor para aquellos países que se encuentran en una etapa temprana del desarrollo; en cambio, tiene un menor efecto para aquellos que han alcanzado altos niveles de desarrollo. 
Figura 1. Esperanza de vida al nacer vs. Gasto total en salud para la ALC y OCDE

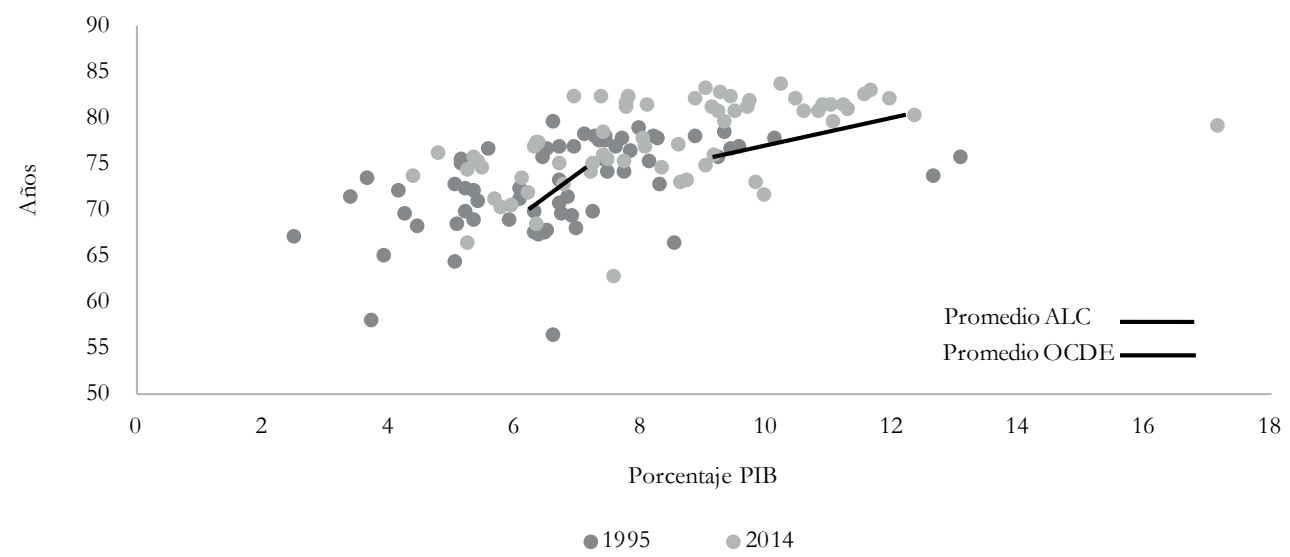

Fuente: elaboración propia a partir de datos del Banco Mundial.

Figura 2. Tasa de mortalidad infantil vs. Gasto total en salud para ALC y OCDE

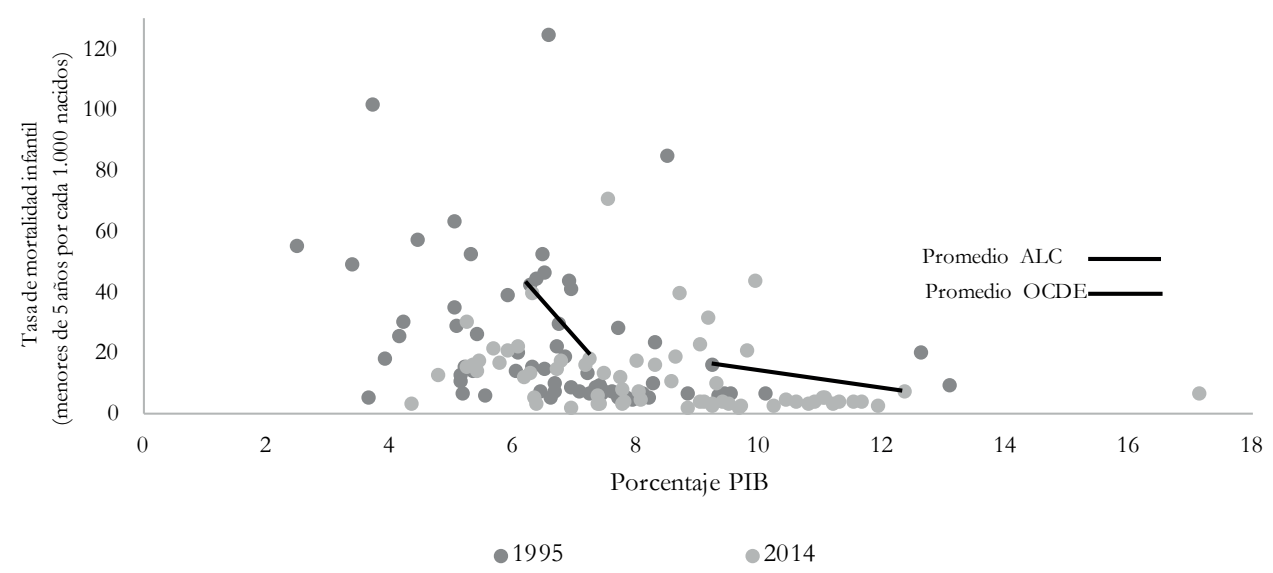

Fuente: elaboración propia a partir de datos del Banco Mundial. 
Sanmartín-Durango et al.: Eficiencia del gasto en salud en la OCDE y ALC...

\section{Metodología y datos}

\section{A. Modelo propuesto}

El DEA ha ganado relevancia en la medición de la eficiencia de los sistemas de salud, debido a que permite evaluar la eficiencia de un conjunto de entidades, denominadas como $\mathrm{DMU}^{2}$. Para ello, usa métodos de programación lineal con los que construye una frontera de producción convexa, en la cual la curva representa las unidades eficientes, y dentro de la misma se encuentran las demás observaciones - entendidas como ineficientes-. De esto último se deriva el término "envolvente", el cual indica que la frontera agrupa todo el conjunto de DMU.

El DEA es un modelo no paramétrico; es decir, que para la construcción del análisis de eficiencia no es necesario tener a priori la especificación de una forma funcional concreta. Para esta metodología es suficiente definir ciertas propiedades formales que debe cumplir el conjunto de datos en evaluación, que hace referencia a los recursos utilizados para producir (insumos) y los resultados obtenidos a partir de esos recursos (productos). Una de las ventajas del DEA es, precisamente, que permite incluir múltiples insumos y productos (Ji \& Lee, 2010).

El modelo construye una razón de valores ponderados que arroja una medida de eficiencia global y proporciona una ordenación de las unidades de decisión con un alcance que no consiguen otros enfoques (Muñoz-Repiso, 2001). Ahora bien, dicha medida de eficiencia puede orientarse tanto a los productos como a los insumos. En el primer caso, el DEA se centra en maximizar los productos manteniendo los insumos constantes, mientras que en el segundo busca minimizar los insumos dejando constantes los productos.

En la literatura se pueden observar dos versiones del DEA. Por un lado, se encuentra el modelo de rendimientos constantes a escala, conocido por las siglas CCR, dado que se deriva de la investigación de Charnes, Cooper y Rhodes (1978), quienes, a su vez, basaron su análisis en el trabajo de Farell (1957). Además del supuesto de convexidad de la frontera de producción, estos autores suponen dos propiedades adicionales: la primera hace referencia

2 Sigla del término en inglés Decision Making Units. 
a la libre disponibilidad de insumos y productos, es decir, que cada DMU puede obtener menos (iguales) resultados con el mismo (mayor) nivel de recursos (Schwartzmann, 2003); y la segunda establece que la producción tiene rendimientos de escala constantes. Por otro lado, se encuentra el modelo de rendimientos variables a escala, conocido como BCC, que es una variación del primero y fue presentado por primera vez por Banker, Charnes y Cooper (1984). Este modelo mantiene los supuestos de convexidad y disponibilidad, pero asegura que la producción tiene rendimientos variables a escala, lo que permite que cada DMU sea comparada con otras de tamaños similares.

La elección, tanto de la versión como de la orientación del modelo, depende del contexto en el que se desenvuelven las DMU analizadas y del objetivo de la investigación. Así, si la metodología es aplicada de manera adecuada, se podrá obtener un indicador de eficiencia, las holguras que señalan las cantidades de insumos y productos a disminuir e incrementar, respectivamente, y los coeficientes que muestran la importancia de cada variable en la determinación de la eficiencia (Muñoz-Repiso, 2001). Todas estas ventajas hacen que el DEA se destaque dentro de los métodos de evaluación de eficiencia. Sin embargo, no se desconoce que presenta limitaciones tales como la posibilidad de clasificar como ineficiente un comportamiento explicado por factores exógenos a las DMU (Bosch et al., 1995); la debilidad para señalar los aspectos concretos que generan la ineficiencia, ya que expone solo los focos potenciales que puedan generarla (Muñoz-Repiso, 2001); finalmente, es necesaria una interpretación exhaustiva del modelo para realizar el diagnóstico.

\section{B. Tratamiento de los datos y elección del modelo}

Para el análisis se utilizaron datos de 62 países, denominados DMU (28 pertenecientes a ALC y 34 a la OCDE); además se incluye el promedio de los mismos en cada grupo como DMU adicionales. Con el fin de hacer una comparación entre ambos grupos, se calculó la eficiencia de cada conjunto de países por separado. La información fue extraída de la base de datos del Banco Mundial, asegurando la comparabilidad, que es un requisito fundamental del DEA. Sin embargo, para el caso de ALC, la falta de uno o de varios datos 
Sanmartín-Durango et al.: Eficiencia del gasto en salud en la OCDE y ALC...

relevantes hizo necesario que se omitieran países como San Vicente y las Granadinas, Antigua y Barbuda, Islas del Carmen, Islas Turcas y Caicos, Puerto Rico y Dominica. Además, fue necesario omitir a Argentina, puesto que la serie histórica del gasto presenta inconsistencias. De esta manera, incluyendo los promedios de ALC y la OCDE como DMU en cada grupo, ALC queda con 30 DMU, mientras que en la OCDE se analizan 36.

Luego de definir los países objeto del análisis, se eligieron los insumos y los productos, basados en la selección de variables de trabajos similares (que se observan en la Tabla 3) y siguiendo el procedimiento sugerido por Cook, Tone y Zhu (2014), el cual está compuesto de las tres fases siguientes:

i. Evaluación de variables. Elaboración de una lista inicial de factores que se pueden considerar recursos y resultados del sistema de salud, con la cual se busca encontrar las diferencias entre los países, para posteriormente depurarlos mediante consideraciones como las de variables conflictivas o confusas, grado de pertinencia y eliminación de datos que repiten información.

ii. Análisis cuantitativo no DEA. En esta fase se debe asegurar que las variables elegidas sean medibles numéricamente, en este caso en función de la caracterización de los sistemas de salud. Si se eligiera un factor meramente cualitativo, es importante localizar una variable mensurable en sustitución.

iii. Análisis basado en DEA. Se procura aplicar la metodología con variables tentativas e identificar cuáles son ponderadas como de bajo impacto dentro de las puntuaciones de eficiencia.

Este proceso dio lugar a la selección de tres determinantes para la evaluación de eficiencia del gasto en salud: esperanza de vida y tasa de mortalidad infantil como variables de salida, y gasto total en salud (\% del PIB) como variable de entrada.

Según el Banco Mundial "la tasa de mortalidad infantil es la probabilidad de que un recién nacido muera antes de cumplir 5 años por cada 1.000 nacidos vivos" (2015). En un número importante de investigaciones esta variable se toma como una aproximación del estado de salud general de 
la población, debido a que es un indicador sensible al desarrollo social y económico (Reidpath \& Allotey, 2003).

Tabla 3. Relación del uso de variables de entrada y de salida en trabajos que han implementado el DEA

\begin{tabular}{|c|c|c|c|}
\hline \multicolumn{2}{|c|}{ Trabajos } & \multicolumn{2}{|c|}{ Variables } \\
\hline Autores & Título & Insumos & Productos \\
\hline \multirow{3}{*}{$\begin{array}{l}\text { Laura Asandului, L., } \\
\text { Roman, M., Fatulescu, } \\
\text { P. (2014) }\end{array}$} & \multirow{3}{*}{$\begin{array}{l}\text { The Efficiency of Healthcare } \\
\text { Systems in Europe: A Data } \\
\text { Envelopment Analysis Approac }\end{array}$} & $\begin{array}{l}\text {-Número de doctores por } \\
\text { cada } 10.000 \text { habitantes. }\end{array}$ & -Esperanza de vida al nacer. \\
\hline & & $\begin{array}{l}\text {-Número de camas por } \\
\text { cada } 10.000 \text { habitantes. }\end{array}$ & $\begin{array}{l}\text {-Esperanza de vida ajustada a } \\
\text { la salud (HALE). }\end{array}$ \\
\hline & & $\begin{array}{l}\text {-Gasto en salud público } \\
\text { (\% PIB). }\end{array}$ & -Tasa de mortalidad infantil. \\
\hline \multirow{4}{*}{$\begin{array}{l}\text { Mirmirani, S., Li, HC., } \\
\text { Ilacqua J. (2011) }\end{array}$} & \multirow{4}{*}{$\begin{array}{l}\text { Health Care Efficiency In } \\
\text { Transition Economies: An } \\
\text { Application Of Data } \\
\text { Envelopment Analysis }\end{array}$} & $\begin{array}{l}\text {-Gasto en salud per } \\
\text { cápita en dólares ajustado } \\
\text { por PPA. }\end{array}$ & $\begin{array}{l}\text {-Esperanza de vida media } \\
\text { para hombres y mujeres. }\end{array}$ \\
\hline & & $\begin{array}{l}\text {-Número de camas por } \\
1.000 \text { habitantes. }\end{array}$ & \multirow{3}{*}{-Tasa de mortalidad infantil. } \\
\hline & & $\begin{array}{l}\text {-Número de médicos por } \\
1.000 \text { habitantes. }\end{array}$ & \\
\hline & & $\begin{array}{l}\text {-Tasa de inmunización } \\
\text { contra el sarampión. }\end{array}$ & \\
\hline \multirow{4}{*}{$\begin{array}{l}\text { Afonso, A. y St Aubyn, } \\
\text { M. (2004) }\end{array}$} & \multirow{4}{*}{$\begin{array}{l}\text { Non-parametric Approaches to } \\
\text { Education and Health } \\
\text { Expenditure Efficiency in } \\
\text { OECD Countries }\end{array}$} & $\begin{array}{l}\text {-Número de médicos por } \\
1.000 \text { habitantes. }\end{array}$ & $\begin{array}{l}\text { - Tasa de mortalidad infantil, } \\
\text { que la convierte a tasa de } \\
\text { supervivencia infantil. }\end{array}$ \\
\hline & & $\begin{array}{l}\text {-Número de enfermeras } \\
\text { por } 1.000 \text { habitantes. }\end{array}$ & \multirow{3}{*}{-Esperanza de vida al nacer. } \\
\hline & & $\begin{array}{l}\text {-Número de camas por } \\
1.000 \text { habitantes }\end{array}$ & \\
\hline & & $\begin{array}{l}\text {-Gasto total en salud } \\
\text { per cápita. }\end{array}$ & \\
\hline
\end{tabular}

Fuente: elaboración propia a partir de la revisión de la literatura.

En este punto, es importante hacer una salvedad: el uso de la tasa de mortalidad infantil como un resultado representa un problema para el DEA, ya que se requiere que las salidas estén expresadas de tal forma que "más sea mejor"; sin embargo, Alfonso y St. Aubyn (2004) resuelven este problema calculando una tasa de supervivencia infantil (TSI), formulada de la siguiente manera: 
Sanmartín-Durango et al.: Eficiencia del gasto en salud en la OCDE y ALC...

$$
T S I=\frac{1000-\text { Tasa de mortalidad infantil }}{\text { Tasa de mortalidad infantil }} .
$$

Esta razón se puede interpretar como la proporción de niños que sobrevivieron a sus primeros 5 años sobre el número de niños que murieron.

Por otra parte, la esperanza de vida representa la cantidad de años que viviría un recién nacido si los patrones de mortalidad vigentes al momento de su nacimiento no cambiaran a lo largo de su vida. De acuerdo con Lago et al. (2013), este indicador refleja con cierto grado de precisión el nivel de salud de una comunidad, pues está asociado directamente con otros determinantes como la tasa de mortalidad de niños, jóvenes y adultos, la efectividad de los sistemas de salud en la atención materna, los niveles de pobreza y otras dimensiones del desarrollo humano.

Por último, el gasto total en salud (\% del PIB) contiene tanto el gasto público como el gasto privado, por lo que, de esta manera, abarca la prestación de servicios de salud (preventivos y curativos), las actividades de planificación familiar, las actividades de nutrición y la asistencia de emergencias de salud. La elección de esta variable como insumo se decidió luego de una discusión sobre si efectivamente representa una medida del esfuerzo sanitario de los países, ya que, si bien se reconoce la limitación de incluir solo el gasto total en salud ( $\%$ PIB) como variable de insumo, es necesario resaltar algunos aspectos. Por una parte, la disponibilidad de datos no permitió incluir algunas variables como tasa de médicos, enfermeras o camas y, por otra parte, en el caso de otras variables de gasto o PIB per cápita, estas tenían mucho ruido, de forma que se generaban resultados sensibles y limitaba el análisis de los mismos.

Una vez definidas las variables, y teniendo en cuenta el objetivo de la investigación, se usa un DEA orientado a los productos, pues se considera que lo mejor para los países es maximizar sus resultados en salud, sin modificar los recursos disponibles. Además, se adopta el supuesto de rendimientos variables a escala, con lo cual se busca coherencia con la teoría en cuanto a que un mayor nivel de gasto implica mejores resultados, pero con un menor ritmo de crecimiento. 
La formalización matemática de la programación lineal resultante se describe de la siguiente forma: se tiene $k=1$ insumos y $m=2$ productos para $n_{1}=30 \mathrm{DMU}$ y $n_{2}=36 \mathrm{DMU}$, de ALC y OCDE, respectivamente. Además, $\boldsymbol{X}$ es la matriz $(k \times n)$ de insumos y $\boldsymbol{Y}$ la matriz $(m \times n)$ de salidas. El problema de programación lineal, para la $i$-ésima DMU, sería entonces:

$$
\begin{gathered}
\operatorname{máx}_{\beta, \lambda} \beta \\
\text { Sujeto a: }-\boldsymbol{X} \lambda+x_{i} \geq 0 \\
\boldsymbol{Y} \lambda-\beta y_{i} \geq 0 \\
N \lambda=\lambda \geq 0
\end{gathered}
$$

$i=1,2,3, \ldots, n_{1}$ o $n_{2}$ (según el grupo de interés.); y $x_{i}$ y $y_{i}$ representan el conjunto de insumos y productos del país $i$.

Así las cosas, $\beta$ es un escalar que se encuentra entre 1 e $\infty$, y su inversa es la puntuación de eficiencia que tiene valores entre 0 y 1 . Su propósito es medir la distancia entre la DMU evaluada y la frontera de eficiencia, definida como la combinación lineal de observaciones con mejores prácticas. Para esta investigación, cuando $\beta<1$, el país en cuestión está dentro de la frontera (es ineficiente), mientras $\beta=1$ implica que está en la frontera (es eficiente).

Además, $\lambda$ es un vector $(n \times 1)$ de constantes que mide los pesos utilizados para calcular la localización de una DMU ineficiente, si llegara a ser eficiente.

La especificación del modelo bajo la hipótesis de retorno variable a escala implica la condición de convexidad de la frontera. Esto supone que la restricción $N_{1} \lambda=1$ debe ser introducida al modelo, donde $N_{1}$ es un vector de 1 de $n$ dimensiones. La ausencia de esta restricción implicaría rendimientos a escala constantes (Muñoz-Repiso, 2001).

Para el cálculo del DEA se usó el software gratuito conocido como DEA Solver-LV Además, reconociendo los cambios continuos de los sistemas de salud, se aplicó la metodología para tres años (1995, 2005 y 2014), con el propósito de identificar tanto las naciones con una eficiencia estable, como las que varían de una década a otra. 
Sanmartín-Durango et al.: Eficiencia del gasto en salud en la OCDE y ALC...

\section{Resultados}

\section{A. Resultados del modelo}

\section{Puntuación y ranking de eficiencia}

En este punto es necesario aclarar que la eficiencia calculada por el DEA es relativa; es decir que la puntuación técnica obtenida por una DMU refleja la eficiencia de ésta en comparación con las demás. Para entender esto, basta con observar los resultados de México y Chile, países que hacen parte de los dos grupos y cuya clasificación es notoriamente mejor cuando se comparan con los países latinoamericanos que cuando se hace con el grupo de la OCDE

En la Tabla 4 se observan los resultados para ALC por el modelo BCC-O ${ }^{3}$, donde, para efectos de la comparación, se incluyó el promedio de la OCDE como un país. Si analizamos el comportamiento de los países eficientes de este grupo, se destaca Cuba como el único que mantiene esta condición en los tres momentos. También se destacan Chile y Jamaica, que cumplen esta cualidad para los dos últimos años, y en 1995 sus puntuaciones son cercanas a uno. Por otro lado, si bien Ecuador es eficiente en 1995, pasa a ocupar el puesto 12 del ranking en 2005 y termina en el puesto 16 en 2014. Este comportamiento es contrario al de República Dominicana y Venezuela, que, después de ocupar los puestos 15 y 12 en 1995, en 2014 son considerados como eficientes.

Ahora, si se dirige la atención a los países ineficientes, en los tres años analizados, 3 de los 30 se mantienen en las últimas posiciones de la clasificación: Guyana, Bolivia y Haití. Esto concuerda con el hecho de que los tres países tienen resultados en salud muy por debajo del promedio, de acuerdo con su nivel de gasto.

Además, se puede notar que el promedio de la OCDE se comporta como un país cercano a la eficiencia en los tres años, lo que refleja el adelanto del grupo económico en materia de desarrollo y le permite tener unas condiciones de eficiencia más estables, como consecuencia de una mejor utilización de los recursos.

3 Rendimientos variables a escala según el enfoque de salida. 
Tabla 4. Resultados del DEA por el modelo BCC-O para ALC, 1995, 2005 y 2014

\begin{tabular}{|c|c|c|c|c|c|c|c|c|}
\hline \multicolumn{3}{|c|}{1995} & \multicolumn{3}{|c|}{2005} & \multicolumn{3}{|c|}{2014} \\
\hline Ranking & DMU (País) & Puntuación & Ranking & DMU (País) & Puntuación & Ranking & DMU (País) & Puntuación \\
\hline 1 & Costa Rica & 1 & 1 & Chile & 1 & 1 & Chile & 1 \\
\hline 2 & Cuba & 1 & 2 & Jamaica & 1 & 2 & $\begin{array}{l}\text { República } \\
\text { Dominicana }\end{array}$ & 1 \\
\hline 3 & Ecuador & 1 & 3 & Cuba & 1 & 3 & Cuba & 1 \\
\hline 4 & Chile & 0,9923 & 4 & $\begin{array}{l}\text { Promedio } \\
\text { OCDE }\end{array}$ & 0,995 & 4 & Venezuela & 1 \\
\hline 5 & $\begin{array}{l}\text { Promedio } \\
\text { OCDE }\end{array}$ & 0,9888 & 5 & Costa Rica & 0,993 & 5 & Jamaica & 1 \\
\hline 6 & Jamaica & 0,9848 & 6 & Venezuela & 0,9803 & 6 & $\begin{array}{l}\text { Promedio } \\
\text { OCDE }\end{array}$ & 0,9921 \\
\hline 7 & Panamá & 0,9682 & 7 & Barbados & 0,9737 & 7 & México & 0,9836 \\
\hline 8 & México & 0,9675 & 8 & México & 0,9732 & 8 & Perú & 0,9797 \\
\hline 9 & Uruguay & 0,9605 & 9 & $\begin{array}{l}\text { República } \\
\text { Dominicana }\end{array}$ & 0,9714 & 9 & Granada & 0,9792 \\
\hline 10 & Barbados & 0,9579 & 10 & Perú & 0,9697 & 10 & Costa Rica & 0,9743 \\
\hline 11 & Belice & 0,95 & 11 & Panamá & 0,9661 & 11 & Panamá & 0,9521 \\
\hline 12 & Venezuela & 0,9377 & 12 & Ecuador & 0,9627 & 12 & Santa Lucía & 0,95 \\
\hline 13 & Santa Lucía & 0,9341 & 13 & Uruguay & 0,9618 & 13 & Uruguay & 0,9446 \\
\hline 14 & Bahamas & 0,9308 & 14 & Bahamas & 0,9542 & 14 & Barbados & 0,9349 \\
\hline 15 & $\begin{array}{l}\text { República } \\
\text { Dominicana }\end{array}$ & 0,924 & 15 & Colombia & 0,9397 & 15 & $\begin{array}{l}\text { Promedio } \\
\text { ALyC }\end{array}$ & 0,9343 \\
\hline 16 & Perú & 0,922 & 16 & Santa Lucía & 0,9388 & 16 & Ecuador & 0,931 \\
\hline 17 & $\begin{array}{l}\text { Promedio } \\
\text { ALyC }\end{array}$ & 0,9118 & 17 & Granada & 0,9356 & 17 & Suriname & 0,9292 \\
\hline 18 & Honduras & 0,9112 & 18 & Belice & 0,9312 & 18 & Bahamas & 0,9243 \\
\hline 19 & $\begin{array}{l}\text { Trinidad y } \\
\text { Tobago }\end{array}$ & 0,9084 & 19 & $\begin{array}{l}\text { Promedio } \\
\text { ALyC }\end{array}$ & 0,9272 & 19 & Colombia & 0,9235 \\
\hline 20 & Colombia & 0,9068 & 20 & Nicaragua & 0,9271 & 20 & Guatemala & 0,9221 \\
\hline 21 & Paraguay & 0,906 & 21 & Paraguay & 0,9209 & 21 & Salvador & 0,9195 \\
\hline 22 & Granada & 0,9054 & 22 & Brasil & 0,9135 & 22 & Nicaragua & 0,9179 \\
\hline 23 & Guatemala & 0,8964 & 23 & Honduras & 0,909 & 23 & $\begin{array}{l}\text { Trinidad y } \\
\text { Tobago }\end{array}$ & 0,913 \\
\hline 24 & Suriname & 0,8857 & 24 & $\begin{array}{l}\text { Trinidad y } \\
\text { Tobago }\end{array}$ & 0,9089 & 24 & Brasil & 0,9129 \\
\hline 25 & Brasil & 0,883 & 25 & Salvador & 0,8924 & 25 & Belice & 0,9123 \\
\hline 26 & Nicaragua & 0,8799 & 26 & Guatemala & 0,8856 & 26 & Honduras & 0,8974 \\
\hline 27 & Salvador & 0,8791 & 27 & Suriname & 0,8755 & 27 & Paraguay & 0,8948 \\
\hline 28 & Guyana & 0,854 & 28 & Guyana & 0,8526 & 28 & Guyana & 0,879 \\
\hline 29 & Bolivia & 0,8035 & 29 & Bolivia & 0,8295 & 29 & Bolivia & 0,8751 \\
\hline 30 & Haití & 0,7351 & 30 & Haití & 0,7986 & 30 & Haití & 0,7751 \\
\hline & romedio & 0,9228 & & romedio & 0,9362 & & romedio & 0,9384 \\
\hline
\end{tabular}

Fuente: elaboración propia.

Lecturas de Economía -Lect. Econ. - No. 91. Medellín, julio-diciembre 2019 
Sanmartín-Durango et al.: Eficiencia del gasto en salud en la OCDE y ALC...

Haciendo el mismo análisis para la OCDE por el modelo BCC-O, en la Tabla 5 puede verse que, dentro de los países eficientes, Japón se mantiene sobre la frontera en los tres momentos, mientras que Luxemburgo, Corea e Islandia son eficientes por lo menos en uno de los momentos, y cuando no lo son, su puntuación es cercana a 1. Llama la atención el comportamiento de Turquía, que de ser eficiente en 1995 pasa a ocupar el lugar número 33 entre 36 países en 2005 y vuelve a ser eficiente en 2014. Igualmente, Estonia tiene un comportamiento llamativo, pues pasa de estar en el último lugar del ranking en 1995 a ser un país eficiente en 2005 y, posteriormente, vuelve a caer al puesto 24 en 2014 .

Por otra parte, en el conjunto de DMU que se encuentran al final de la lista, también hay naciones que se repiten para los tres años, como Hungría, República Eslovaca y el promedio de ALC. Esta última DMU merece una especial atención, porque, igual que ocurre en el análisis anterior, el hecho de que el promedio de ALC se encuentre siempre en las últimas posiciones confirma las diferencias de ambos grupos, donde el grupo de la OCDE resulta ser el más favorecido.

\section{Ponderaciones de las variables}

Se observaron las ponderaciones virtuales que el DEA asignó tanto a la entrada como a las salidas en el cálculo de las puntuaciones de eficiencia, con el fin de identificar la participación porcentual de cada una de estas ponderaciones en dicho cálculo.

En el caso de ALC se puede identificar que, para 1995, la eficiencia de Ecuador y Cuba se debió en gran medida a sus resultados en la esperanza de vida y la mortalidad infantil, mientras que la eficiencia en Costa Rica se le puede atribuir en parte al nivel de gasto. En los años siguientes, los países eficientes deben su ubicación en el ranking DEA más a los resultados, con excepción de Cuba, que debe su posición a su nivel de gasto.

Para la OCDE, la mayoría de los países eficientes deben su nivel de eficiencia exclusivamente a los resultados en salud que obtuvieron en los tres años de análisis. Sin embargo, Japón e Islandia son los únicos países donde se pondera más el gasto dentro de la eficiencia. 
Tabla 5. Resultados del DEA por el modelo BCC-O para la OCDE, 1995, 2005 y 2014

\begin{tabular}{|c|c|c|c|c|c|c|c|c|}
\hline \multicolumn{3}{|c|}{1995} & \multicolumn{3}{|c|}{2005} & \multicolumn{3}{|c|}{2014} \\
\hline \multicolumn{3}{|c|}{ Modelo BCC-O } & \multicolumn{3}{|c|}{ Modelo BCC-O } & \multicolumn{3}{|c|}{ Modelo BCC-O } \\
\hline Rank & DMU (País) & Puntuación & Rank & DMU (País) & Puntuación & Rank & DMU (País) & Puntuación \\
\hline 1 & Japón & 1 & 1 & Estonia & 1 & 1 & Japón & 1 \\
\hline 2 & República de Corea & 1 & 2 & Islandia & 1 & 2 & Luxemburgo & 1 \\
\hline 3 & Suecia & 1 & 3 & Japón & 1 & 3 & Turquía & 1 \\
\hline 4 & Turquía & 1 & 4 & República de Corea & 1 & 4 & España & 0,9999 \\
\hline 5 & Luxemburgo & 0,9893 & 5 & Luxemburgo & 1 & 5 & República de Corea & 0,9971 \\
\hline 6 & Islandia & 0,9863 & 6 & Chile & 0,9916 & 6 & Islandia & 0,9957 \\
\hline 7 & Suiza & 0,9859 & 7 & Israel & 0,9894 & 7 & Israel & 0,9949 \\
\hline 8 & Italia & 0,9828 & 8 & Australia & 0,9868 & 8 & Italia & 0,9941 \\
\hline 9 & Canadá & 0,9804 & 9 & Italia & 0,986 & 9 & Suecia & 0,9911 \\
\hline 10 & España & 0,9804 & 10 & Suecia & 0,9843 & 10 & Australia & 0,988 \\
\hline 11 & Australia & 0,9785 & 11 & Chile & 0,9817 & 11 & Chile & 0,9871 \\
\hline 12 & Chile & 0,9785 & 12 & Canadá & 0,9801 & 12 & Francia & 0,9855 \\
\hline 13 & Noruega & 0,9785 & 13 & España & 0,9795 & 13 & Grecia & 0,983 \\
\hline 14 & Francia & 0,9775 & 14 & Francia & 0,9785 & 14 & Irlandia & 0,9829 \\
\hline 15 & Grecia & 0,9755 & 15 & Irlandia & 0,977 & 15 & Canadá & 0,9805 \\
\hline 16 & Israel & 0,9738 & 16 & Noruega & 0,977 & 16 & Noruega & 0,9805 \\
\hline 17 & Países Bajos & 0,9732 & 17 & Nueva Zelanda & 0,9747 & 17 & Suiza & 0,9805 \\
\hline 18 & Finlandia & 0,9675 & 18 & Países Bajos & 0,9685 & 18 & Finlandia & 0,9784 \\
\hline 19 & Bélgica & 0,9661 & 19 & Austria & 0,9683 & 19 & Reino Unido & 0,9752 \\
\hline 20 & Reino Unido & 0,966 & 20 & Grecia & 0,9672 & 20 & Nueva Zelanda & 0,9739 \\
\hline 21 & Nueva Zelanda & 0,9648 & 21 & Reino Unido & 0,9649 & 21 & Austria & 0,9731 \\
\hline 22 & Austria & 0,9639 & 22 & Bélgica & 0,964 & 22 & Países Bajos & 0,9727 \\
\hline 23 & Alemania & 0,9608 & 23 & Alemania & 0,9635 & 23 & Polonia & 0,9719 \\
\hline 24 & Irlandia & 0,9547 & 24 & Finlandia & 0,9624 & 24 & Estonia & 0,97 \\
\hline 25 & México & 0,9537 & 25 & Promedio OCDE & 0,9551 & 25 & Portugal & 0,9692 \\
\hline 26 & Promedio OCDE & 0,9518 & 26 & Portugal & 0,9529 & 26 & México & 0,9682 \\
\hline 27 & Estados Unidos & 0,9508 & 27 & Dinamarca & 0,9502 & 27 & Eslovenia & 0,9681 \\
\hline 28 & Portugal & 0,9469 & 28 & México & 0,9492 & 28 & Alemania & 0,9672 \\
\hline 29 & Dinamarca & 0,9456 & 29 & Eslovenia & 0,9473 & 29 & Bélgica & 0,9641 \\
\hline 30 & Polonia & 0,9349 & 30 & Estados Unidos & 0,9458 & 30 & Dinamarca & 0,9636 \\
\hline 31 & Eslovenia & 0,9299 & 31 & República Checa & 0,9443 & 31 & Promedio OCDE & 0,9586 \\
\hline 32 & República Eslovaca & 0,9219 & 32 & Polonia & 0,9433 & 32 & República Checa & 0,9499 \\
\hline 33 & República Checa & 0,9188 & 33 & Turquía & 0,922 & 33 & Estados Unidos & 0,9444 \\
\hline 34 & Promedio ALyC & 0,8831 & 34 & República Eslovaca & 0,9177 & 34 & República Eslovaca & 0,9279 \\
\hline 35 & Hungría & 0,8775 & 35 & Promedio ALyC & 0,9086 & 35 & Hungría & 0,9208 \\
\hline 36 & Estonia & 0,856 & 36 & Hungría & 0,8868 & 36 & Promedio ALyC & 0,9102 \\
\hline & Promedio & 0,9599 & & Promedio & 0,9658 & & Promedio & 0,9741 \\
\hline
\end{tabular}

Fuente: elaboración propia.

Lecturas de Economía -Lect. Econ. - No. 91. Medellín, julio-diciembre 2019 
Sanmartín-Durango et al.: Eficiencia del gasto en salud en la OCDE y ALC...

\section{Proyecciones}

Para cada año de análisis el modelo realiza proyecciones que le permiten establecer la proporción en la cual los países ineficientes deberían aumentar (reducir) el nivel de gasto, la esperanza de vida al nacer y/o la tasa de supervivencia infantil para eliminar dicha ineficiencia y así alcanzar los niveles que lograron las DMU eficientes en ese periodo, las cuales sirven de referencia para las demás.

Se toman los 5 países más ineficientes en el año 2014 para cada grupo y se observa lo que el modelo les propone aumentar o disminuir para alcanzar el nivel de eficiencia 1. En el caso de los insumos, aunque el modelo está enfocado a los productos, las proyecciones permiten mostrar que, para el caso de ALC, este pide mantener constante el gasto en la mayoría de los casos, lo que implicaría hacer un mejor uso de los recursos. En ese sentido, cabe destacar el caso de Uruguay como el único país que debería reducir el gasto en salud (aproximadamente $3 \%$ ), ya que, en comparación con las demás DMU, es un país que tiene alto gasto, pero no lo refleja en los resultados en salud. Además, tomando como referencia los países eficientes, el DEA propone a todos los países de ALC mejorar los resultados en salud, como es el caso de Guyana, Bolivia y Haití, siendo este último el país que necesita aumentar en gran medida sus resultados en salud, particularmente la tasa de supervivencia infantil.

En el grupo de la OCDE, con relación a los productos, las proyecciones muestran que es mayor el porcentaje y la cantidad de países a los que se les pide reducir el gasto en salud. Cabe resaltar el caso de Estados Unidos, el cual, reduciendo el gasto en un 6,7\%, aproximadamente, podría obtener los mismos resultados con menos recursos en comparación con su gasto actual.

En cuanto a las proyecciones de resultados, se puede observar que para la OCDE el porcentaje de la esperanza de vida que se pide aumentar es menor que el de la región latinoamericana, mientras que debe aumentar en mayor medida la tasa de supervivencia infantil, que se puede explicar, entre otras cosas, por los valores de referencia que imponen las DMU eficientes en cada grupo de países. Por su parte, países como República Checa, República Eslovaca, Turquía, Luxemburgo, México, Polonia, Estonia y Chile deberían 
concentrar sus esfuerzos en aumentar la tasa de supervivencia infantil, pero manteniendo su nivel de gasto constante.

\section{B. Frontera de posibilidades de producción (FPP)}

En el contexto del presente trabajo, se construyó una FPP que representa la eficiencia de los países evaluados en términos del gasto y los resultados en salud para 2014. Los países identificados como eficientes por el modelo, naturalmente, se encuentran ubicados sobre la FPP, lo que evidencia que, en el 2014, presentaron la mejor combinación (en términos de eficiencia); en cambio, aquellos que más alejados se encuentren de la FPP resultan ser los países situados en los últimos lugares del ranking del modelo. En la Figura 3 se presenta el resultado de la FPP para los países de ALC así como para el promedio de la OCDE.

De esta manera, para ALC, la FPP ubica sobre la frontera aquellos países cuyo nivel de eficiencia resulta ser 1 para el 2014: Cuba, Chile, Venezuela y República Dominicana. Respecto a este último, cabe anotar que, por defecto, al tratarse del país con el menor nivel de gasto en salud para ese año, es calificado por el modelo ${ }^{4}$ como un país eficiente, mientras que los demás resultan ser los países que efectivamente alcanzaron la máxima eficiencia relativa, exceptuando a Jamaica. Este caso en particular no está situado sobre la FPP pero sí muy cerca de ella; la razón es que, de acuerdo con el modelo, existe alguna diferencia entre las variables observadas y la proyección que el mismo modelo realiza como faltante para alcanzar esta eficiencia. Tal diferencia, que es de 0,04 y 0,03 puntos básicos para la esperanza de vida al nacer y la tasa de supervivencia infantil, respectivamente, implica que, en la figura, el país no logre ubicarse exactamente sobre la frontera. Entre tanto, los países que ocuparon los últimos lugares corresponden a aquellas DMU que están más alejadas de la FPP.

4 En el modelo por rendimientos variables de escala mediante el enfoque de maximización de salidas (DEA BCC-O), la formulación matemática tiene una restricción donde $\lambda=1$, por lo tanto, el modelo DEA toma por defecto la DMU con la menor entrada y la DMU con la mayor salida como eficientes para cumplir dicha restricción. 
Sanmartín-Durango et al.: Eficiencia del gasto en salud en la OCDE y ALC...

Figura 3. FPP como combinación del gasto y los resultados en salud para ALC, $2014^{5}$

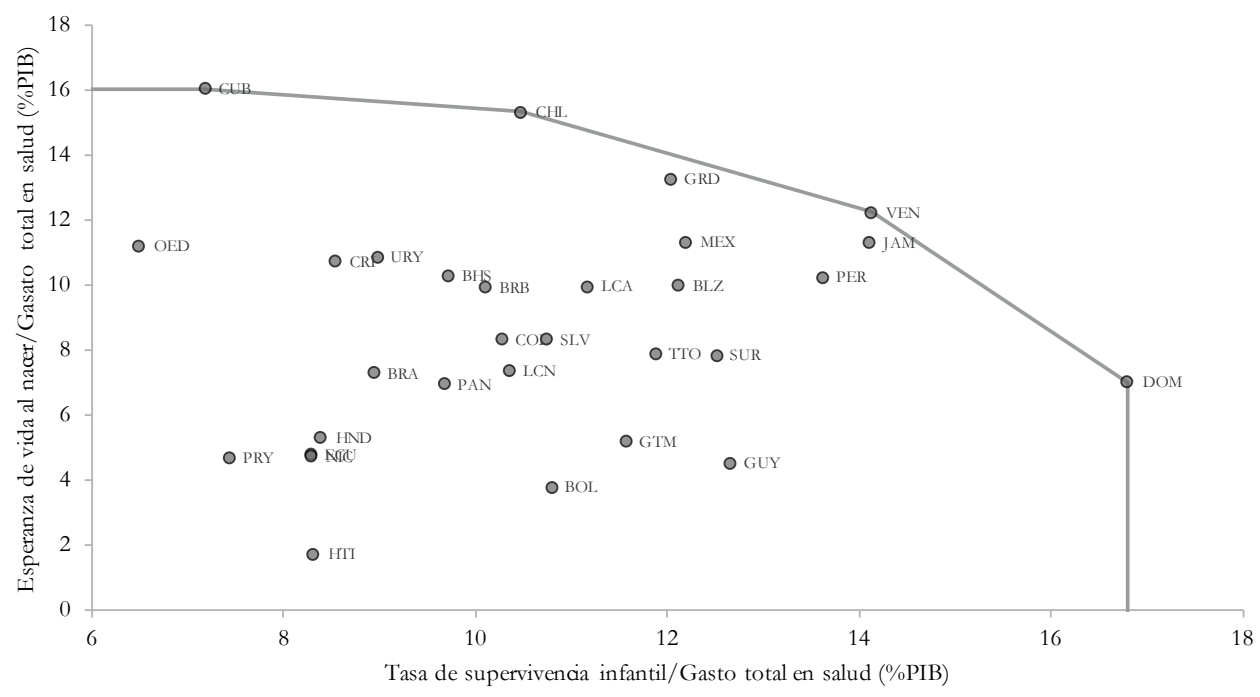

Fuente: elaboración propia.

Por su parte, en el caso de la OCDE, los países que conformaron la FPP del 2014 en la Figura 4 son Luxemburgo, Estonia y Turquía, siendo éste último aquél país con el menor gasto en salud dentro del grupo económico para ese año. Si bien Japón fue calificado con una eficiencia relativa de uno, no es situado sobre la frontera, en tanto presenta una diferencia entre el dato observado y el proyectado por el modelo para alcanzar la mayor eficiencia de 0,0008 y 0,0080 en las variables esperanza de vida y tasa de supervivencia infantil respectivamente. En esta ocasión, resulta destacar, lo distante que se encuentra Estados Unidos de la eficiencia en el gasto en salud, corroborando

5 Las abreviaturas de la figura 3 corresponden a los siguientes países: Bahamas (BHS), Belice (BLZ), Bolivia (BOL), Brasil (BRA), Barbados (BRB), Chile (CHL), Colombia (COL), Costa Rica (CRI), Cuba (CUB), República Dominicana (DOM), Ecuador (ECU), Granada (GRD), Guatemala (GTM), Guyana (GUY), Honduras (HND), Haití (HTI), Jamaica (JAM), Santa Lucía (LCA), México (MEX), Nicaragua (NIC), Panamá (PAN), Perú (PER), Paraguay (PRY), El Salvador (SLV), Suriname (SUR), Trinidad y Tobago (TTO), Uruguay (URY) y Venezuela (VEN). 
la hipótesis que el país americano presenta un gasto excesivo para el nivel de resultados en salud en el que se ubica.

Figura 4. FPP como combinación del gasto y los resultados en salud para la OCDE, $2014^{6}$

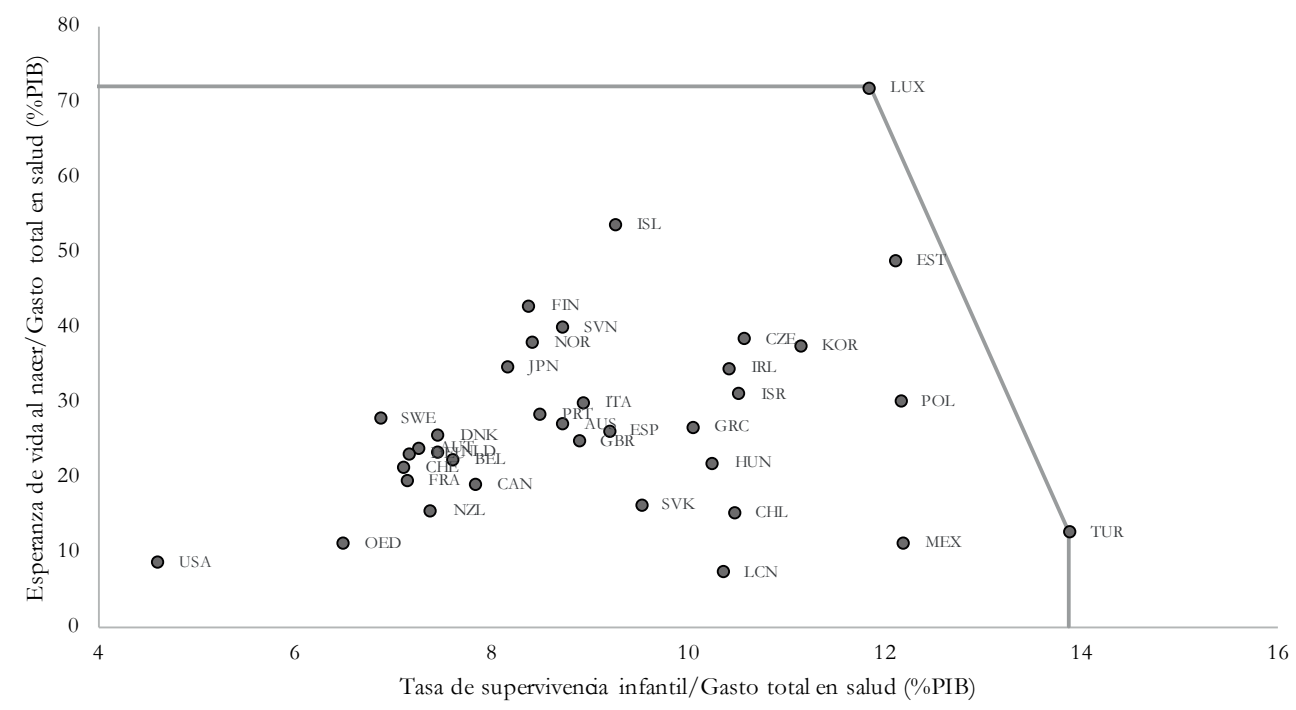

Fuente: elaboración propia.

\section{Comparación de resultados}

Debido a que existen trabajos que han aplicado la metodología DEA u otras metodologías de técnicas no paramétricas para medir la eficiencia en salud, es posible hacer una comparación de los resultados con dichos trabajos

$6 \quad$ Las abreviaturas de la figura 4 corresponden a los siguientes países: Australia (AUS), Austria (AUT), Bélgica (BEL), Canadá (CAN), Suiza (CHE), Chile (CHL), República Checa (CZE), Alemania (DEU), Dinamarca (DNK), España (ESP), Estonia (EST), Finlandia (FIN), Francia (FRA), Reino Unido (GBR), Grecia (GRC), Hungría (HUN), Irlanda (IRL), Islandia (ISL), Israel (ISR), Italia (ITA), República de Corea (KOR), Luxemburgo (LUX), México (MEX), Países Bajos (NLD), Noruega (NOR), Nueva Zelanda (NZL), Polonia (POL), Portugal (PRT), República Eslovaca (SVK), Eslovenia (SVN), Suecia (SWE), Turquía (TUR) y Estados Unidos (USA). 
Sanmartín-Durango et al.: Eficiencia del gasto en salud en la OCDE y ALC...

para identificar coincidencias y diferencias, y para corroborar la importancia del método aplicado.

De esta forma, para ALC, los resultados del modelo coinciden con algunos de los obtenidos por Machado (2006) en el modelo $\mathrm{FDH}^{7}$ orientado al uso del gasto, ya que este autor analiza, para el periodo 1990-2003, algunos de los países que se encuentran en el grupo latinoamericano. Los resultados coinciden en que países como Chile, Ecuador y Costa Rica son eficientes, mientras que Venezuela, Uruguay y México tienen una puntuación muy cercana a 1. Además, afirma que países como Bolivia y Nicaragua se encuentran en los últimos lugares del ranking. Sin embargo, baja este método, Colombia se encuentra mejor ubicado que en los resultados obtenidos mediante el DEA.

Por otra parte, en el grupo de la OCDE, los resultados obtenidos se pueden confirmar con los derivados del trabajo de Alfonso y St. Aubyn (2004), donde se realiza un análisis de FDH y DEA por BCC orientado a productos y variables de insumos similares; además, el grupo de países es el mismo (excepto Chile). En los resultados, los autores encuentran que tanto los países eficientes como los ineficientes en el modelo DEA por BCCO coinciden con los de este modelo, en el cual Islandia, Japón, República de Corea, Luxemburgo y Turquía hacen parte de los eficientes y Hungría, Estados Unidos, Irlanda y Portugal son los más alejados del nivel de eficiencia.

Lo anterior permite concluir que la aplicación de la metodología DEA, teniendo en cuenta los requisitos y límites de la misma, es adecuada para medir la eficiencia de los países tanto en el sector salud como en otros sectores. Además, las coincidencias encontradas con otros trabajos similares dan solidez y soporte a los resultados del modelo propuesto para cuantificar la eficiencia del gasto en salud de los países de ALC y la OCDE.

Sigla del término en inglés Free Disposable Hull. 


\section{Análisis de sensibilidad}

\section{Promedios quinquenales con rezago en la variable explicativa}

Se realizó un nuevo ejercicio para ambos grupos de países usando promedios quinquenales de las variables. De esta forma, el período 1995-2014 se dividió en 4 quinquenios y el modelo DEA se corrió para los quinquenios 2000-2004, 2005-2009 y 2010-2014 con la variable del gasto total rezagada un quinquenio. El objetivo de este análisis está apoyado en el argumento de que el gasto en salud de un año difícilmente variará la esperanza de vida o la mortalidad infantil de ese año. Para poder hacer una comparación con el modelo original el modelo DEA se adopta nuevamente el supuesto de rendimientos variables a escala orientado a los productos.

Los resultados siguen siendo similares a los obtenidos en el modelo principal. En el caso del grupo de ALC se observa que el ranking se comporta de manera similar: Cuba sigue conservando la eficiencia en todos los períodos, mientras que Haití, Bolivia y Guyana siguen conservando los últimos lugares del ranking. Sin embargo, Chile se convierte en un país eficiente en todos los períodos; se eliminan los cambios atípicos en la eficiencia de Ecuador; Jamaica muestra ahora un comportamiento atípico, ya que pasa de ser ineficiente en los períodos 2000-2004 y 2005-2009 a ser eficiente en el período 2010-2014; Belice pasa de estar muy lejos de la eficiencia en el modelo principal a ser eficiente en los dos primeros períodos de este modelo e ineficiente en el último; y Venezuela no es eficiente en ningún periodo a diferencia del modelo anterior. Los resultados de este ejercicio se encuentran en la Tabla $1 \mathrm{~A}$ de los anexos.

Para el caso de la OCDE, Japón y República de Corea se comportan de forma eficiente para todos los períodos, pero Luxemburgo e Islandia se vuelven eficientes en todos los períodos, a diferencia del modelo anterior, en el cual ambos se encontraban cerca de la eficiencia en 1995, pero no tenían la puntuación de 1. Por otra parte, se observa que se mantiene el comportamiento atípico de países como Suecia, Turquía y Estonia. En cuanto a los países que se encontraban al final del ranking como los menos eficientes, se observa que siguen siendo Hungría, República Eslovaca y el promedio de 
Sanmartín-Durango et al.: Eficiencia del gasto en salud en la OCDE y ALC...

ALC. Los resultados de este ejercicio se encuentran en la Tabla 2A de los anexos.

\section{Modelo DEA con todos los paises}

En el ejercicio de unir ambos grupos de países en uno solo, el modelo DEA, orientado a los productos bajo el supuesto de rendimientos variables a escala, arroja resultados muy similares a los que se tenía con ambos grupos por separado, ya que los países eficientes en este modelo son los países eficientes en los modelos DEA realizados anteriormente. Sin embargo, se observa que, en los tres años, son más eficientes los países que pertenecen al grupo OCDE que a la región latinoamericana.

La mayoría de los países OCDE tienen índices de eficiencia muy cercanos a 1, mientras que en el grupo de ALC la mayoría se encuentran en las últimas posiciones del ranking y, por tanto, tienen índices de eficiencia bajos; esto puede ser explicado por el concepto de eficiencia relativa. Del grupo ALC, se destacan países como Ecuador, Cuba, Chile, República Dominicana, Jamaica, Costa Rica y Venezuela, que, en el ranking, todos los años se encuentran por debajo del puesto 25 de 62 DMU. Por su parte, en el grupo OCDE, países como Hungría y Estonia se encuentran en posiciones por encima del lugar 50 y con un índice de eficiencia relativamente bajo. La tabla de resultados de este ejercicio se encuentra en la Tabla 2A de los anexos.

\section{Conclusiones}

Con respecto al análisis descriptivo puede resaltarse, en primer lugar, que las condiciones de salud a nivel mundial han mejorado significativamente, puesto que se vive más y mejor. Por ejemplo, en el periodo 1995-2014 se registró un aumento de 5 años en la esperanza de vida -al pasar esta de 66 a 71 años-, una reducción de la tasa de mortalidad infantil de 153 niños por cada 1.000 nacidos vivos y una disminución en la tasa de mortalidad materna de 178 muertes por cada 100.000 nacidos. Si bien estos resultados no reflejan las particularidades de los países, el común denominador es una evolución positiva del desempeño de las principales variables de resultados en salud, que 
apuntan a su mejoramiento continuo. Esta transición que no ha sido ajena a la región de ALC y a los países miembros de la OCDE.

En ese mismo sentido, pese a las diferencias en los recursos que destinan las naciones para atender sus necesidades en salud, existe un incremento notable en respuesta a la presión que se deriva tanto de la oferta como de la demanda. Sin embargo, en los últimos años, el ritmo de crecimiento del gasto en salud realizado por ALC es mayor que el de la OCDE, debido a un mayor margen de maniobra de la región latinoamericana, como resultado de una etapa más temprana del nivel de desarrollo. Esto supone, entre otras cosas, una convergencia entre ambos grupos de países en el mediano plazo.

De esta forma, los hechos estilizados permiten concluir que un mayor gasto en salud no refleja mejores resultados; es decir, el país que más gasta no necesariamente es el que obtiene el balance más destacado en las condiciones de salud.

Por otra parte, según la proyección del DEA, los resultados deben aumentarse en mayor proporción por los países de ALC que por los países miembros de la OCDE, dados los niveles de desarrollo de este último grupo. Además, para casos como Colombia, Perú, Brasil y Costa Rica - países con intención de ser miembros del grupo OCDE, o candidatos a serlo-, se puede deducir que deberían contener su nivel de gasto, haciendo un uso óptimo del mismo e incrementando sus niveles de resultados, en promedio, en $4,4 \%$ para la esperanza de vida y 33,3\% para la tasa de supervivencia infantil, aproximadamente. Con esto, es necesario destacar, en ambos grupos, casos como los de Uruguay y Estados Unidos, países que confirman el hecho de que gastar más no necesariamente es gastar mejor, pues son aquellos a los que el modelo proyecta la mayor reducción del gasto en salud (en promedio 3,7\% para Uruguay y 6,7 \% para Estados Unidos). Además, los resultados arrojados por el modelo para cada grupo concuerdan con el enfoque de maximización de las salidas, propuesto inicialmente.

En cuanto al análisis de sensibilidad, se observa que los principales resultados del modelo se mantienen ante variaciones como la unión de ambos grupos de países y los promedios quinquenales con rezago en la variable 
Sanmartín-Durango et al.: Eficiencia del gasto en salud en la OCDE y ALC...

explicativa, a excepción de países como Belice, Luxemburgo e Islandia, los cuales pasan a ser eficientes (puntuación 1) en algún periodo de tiempo.

Finalmente, este trabajo resulta relevante en la medida en que permite a los hacedores de política orientar el esfuerzo fiscal hacia un mejor gasto en salud, sin que ello implique el aumento del mismo. No obstante, se reconoce la carencia de la disponibilidad y calidad de la información y cómo estas condiciones limitan los resultados obtenidos, especialmente por el hecho de que la inclusión de más variables de insumos podría explicar los resultados que se obtienen en salud. Así mismo, es importante precisar que, dado que este análisis se encuentra en el marco de la eficiencia relativa, la adición de nuevos insumos, productos o DMU implicaría modificaciones en los resultados.

Estas conclusiones plantean la necesidad de continuar la investigación en torno a tres aspectos claves: el primero, es un análisis de los sistemas sanitarios a nivel micro, enfocado en la manera como se utilizan los recursos destinados a la salud. El segundo aspecto es un análisis DEA en series de tiempo, lo que permitiría ampliar la visión de la eficiencia hacia escenarios más dinámicos. Esto, reconociendo que es un desarrollo reciente de la metodología. Finalmente, sería conveniente hacer uso del método de frontera estocástica, dado que es una medida paramétrica que además de arrojar un índice de eficiencia relativa permite identificar el origen de la ineficiencia.

\section{Anexos}

A continuación, se presentan los modelos DEA por rendimientos variables a escala según el enfoque de salidas para cada grupo y aplicando promedios quinquenales de rezago en la variable explicativa (Tablas 1A y 2A). Además, se incluye el modelo DEA por rendimientos variables a escala según el enfoque de salidas en promedios quinquenales para ambos grupos de países presentados como un solo grupo de análisis (Tabla 3A). 
Tabla 1A. Modelo DEA para ALC

\begin{tabular}{|c|c|c|c|c|c|c|c|}
\hline \multirow{2}{*}{$\mathrm{DMU}$} & \multicolumn{2}{|c|}{$2000-2004$} & \multicolumn{2}{|c|}{ 2005-2009 } & \multicolumn{2}{|c|}{ 2010-2014 } & \multirow{2}{*}{ DMU's de referencia } \\
\hline & Puntuación & Ranking & Puntuación & Ranking & Puntuación & Ranking & \\
\hline Bahamas & 0,9386 & 14 & 0,9734 & 11 & 0,9261 & 15 & Costa Rica, Chile, Cuba, Ecuador \\
\hline Belice & 1 & 1 & 1 & 1 & 0,9232 & 17 & Chile, Jamaica, Belice \\
\hline Bolivia & 0,8175 & 29 & 0,8391 & 29 & 0,8763 & 28 & Cuba, Ecuador, Chile, Jamaica \\
\hline Brasil & 0,9114 & 21 & 0,9131 & 21 & 0,9128 & 22 & Costa Rica, Ecuador, Cuba, Chile \\
\hline Barbados & 0,9653 & 10 & 0,9725 & 12 & 0,9633 & 11 & Costa Rica, Cuba, Chile, Jamaica, Rep. Domin. \\
\hline Chile & 1 & 1 & 1 & 1 & 1 & 1 & Chile \\
\hline Colombia & 0,9204 & 20 & 0,9442 & 18 & 0,9212 & 18 & Costa Rica, Chile, Jamaica, Ecuador \\
\hline Costa Rica & 1 & 1 & 0,9877 & 10 & 0,9774 & 8 & Costa Rica, Chile \\
\hline Cuba & 1 & 1 & 1 & 1 & 1 & 1 & Cuba \\
\hline Rep. Domin. & 0,9263 & 17 & 0,9493 & 15 & 1 & 1 & Cuba, Ecuador, Rep. Domin. \\
\hline Ecuador & 1 & 1 & 1 & 1 & 0,964 & 10 & Ecuador, Chile \\
\hline Granada & 0,9098 & 24 & 0,9127 & 22 & 0,9294 & 14 & Costa Rica, Chile, Cuba, Jamaica \\
\hline Guatemala & 0,9255 & 18 & 0,8894 & 26 & 0,8808 & 27 & Cuba, Ecuador, Chile \\
\hline Guyana & 0,8568 & 28 & 0,852 & 28 & 0,8289 & 29 & Cuba, Ecuador, Chile, Jamaica \\
\hline Honduras & 0,9223 & 19 & 0,906 & 23 & 0,8995 & 23 & Cuba, Chile \\
\hline Haití & 0,7501 & 30 & 0,7836 & 30 & 0,7962 & 30 & Costa Rica, Chile, Cuba, Ecuador, Jamaica \\
\hline Jamaica & 0,9537 & 12 & 0,9885 & 8 & 1 & 1 & Cuba, Ecuador, Belice, Jamaica \\
\hline Santa Lucía & 0,9275 & 16 & 0,9468 & 16 & 0,9243 & 16 & Costa Rica, Chile, Cuba, Ecuador \\
\hline Prom. ALC & 0,9287 & 15 & 0,9332 & 20 & 0,9209 & 19 & Chile, Costa Rica, Cuba \\
\hline México & 0,9863 & 7 & 0,9881 & 9 & 0,9648 & 9 & Cuba, Ecuador, Chile Jamaica \\
\hline Nicaragua & 0,9102 & 23 & 0,9441 & 19 & 0,9194 & 20 & Chile, Cuba, Ecuador, Jamaica \\
\hline Prom. OCDE & 0,9983 & 6 & 0,993 & 7 & 0,9915 & 5 & Costa Rica, Chile, Cuba \\
\hline Panamá & 0,9711 & 8 & 0,9623 & 13 & 0,9548 & 12 & Costa Rica, Chile \\
\hline Perú & 0,9481 & 13 & 0,9963 & 6 & 0,9828 & 6 & Cuba, Ecuador, Belice, Jamaica \\
\hline Paraguay & 0,9093 & 25 & 0,904 & 24 & 0,8979 & 24 & Costa Rica, Chile \\
\hline El Salvador & 0,8918 & 26 & 0,8925 & 25 & 0,8944 & 25 & Costa Rica, Chile, Jamaica \\
\hline Suriname & 0,8777 & 27 & 0,876 & 27 & 0,8909 & 26 & Costa Rica, Chile, Jamaica \\
\hline Trin. y Tob. & 0,9111 & 22 & 0,9461 & 17 & 0,9193 & 21 & Cuba, Ecuador, Belice, Chile, Jamaica \\
\hline Uruguay & 0,9663 & 9 & 0,9576 & 14 & 0,9481 & 13 & Costa Rica, Chile \\
\hline Venezuela & 0,9631 & 11 & 0,9964 & 5 & 0,9825 & 7 & Cubam. Ecuador, Belice, Chile, Jamaica \\
\hline Promedio & 0,933 & & 0,942 & & 0,933 & & \\
\hline
\end{tabular}

Nota: resultados DEA ajustado por quinquenios y rezago del gasto total en salud por BCC-O.

Fuente: elaboración propia. 
Sanmartín-Durango et al.: Eficiencia del gasto en salud en la OCDE y ALC...

Tabla 2A. Modelo DEA para OCDE

\begin{tabular}{|c|c|c|c|c|c|c|c|}
\hline \multirow{2}{*}{ DMU } & \multicolumn{2}{|c|}{$2000-2004$} & \multicolumn{2}{|c|}{$2005-2009$} & \multicolumn{2}{|c|}{$2010-2014$} & \multirow{2}{*}{ DMU's de referencia } \\
\hline & Puntuación & Ranking & Puntuación & Ranking & Puntuación & Ranking & \\
\hline Australia & 0,980 & 9 & 0,985 & 7 & 0,987 & 12 & Japón \\
\hline Austria & 0,964 & 17 & 0,971 & 18 & 0,975 & 20 & Japón \\
\hline Bélgica & 0,958 & 24 & 0,965 & 22 & 0,968 & 24 & Japón \\
\hline Canadá & 0,977 & 10 & 0,978 & 12 & 0,982 & 14 & Japón \\
\hline Suiza & 0,985 & 7 & 0,991 & 5 & 0,995 & 6 & Japón \\
\hline Chile & 0,970 & 14 & 0,975 & 15 & 0,989 & 10 & Japón, Rep. Corea \\
\hline Rep. Checa & 0,930 & 32 & 0,942 & 29 & 0,951 & 29 & Japón, Rep. Corea \\
\hline Alemania & 0,960 & 23 & 0,963 & 23 & 0,971 & 23 & Japón \\
\hline Dinamarca & 0,944 & 28 & 0,949 & 27 & 0,962 & 27 & Japón \\
\hline España & 0,974 & 12 & 0,985 & 9 & 0,993 & 8 & Japón, Rep. Corea \\
\hline Estonia & 0,883 & 36 & 0,9203 & 33 & 1 & 1 & Japón, Rep. Corea, Estonia \\
\hline Finlandia & 0,962 & 21 & 0,971 & 17 & 0,976 & 19 & Japón, Rep. Corea, Islandia, Lux \\
\hline Francia & 0,973 & 13 & 0,982 & 11 & 0,988 & 11 & Japón \\
\hline Reino Unido & 0,9623 & 20 & 0,968 & 20 & 0,973 & 21 & Japón, Rep. Corea \\
\hline Grecia & 0,963 & 19 & 0,966 & 21 & 0,973 & 21 & Japón \\
\hline Hungría & 0,885 & 35 & 0,891 & 36 & 0,910 & 35 & Japón, Rep. Corea \\
\hline Irlanda & 0,964 & 18 & 0,978 & 13 & 0,978 & 16 & Japón, Rep. Corea \\
\hline Islandia & 1 & 1 & 1 & 1 & 1 & 1 & Islandia \\
\hline Israel & 0,975 & 11 & 0,984 & 10 & 0,994 & 7 & Japón, Rep. Corea \\
\hline Italia & 0,983 & 8 & 0,986 & 6 & 0,991 & 9 & Japón \\
\hline Japón & 1 & 1 & 1 & 1 & 1 & 1 & Japón \\
\hline Rep. Corea & 1 & 1 & 1 & 1 & 1,000 & 1 & Rep. Corea \\
\hline Prom. ALC & 0,893 & 34 & 0,906 & 35 & 0,908 & 36 & Japón, Rep. Corea \\
\hline Luxemburgo & 1 & 1 & 1 & 1 & 1 & 1 & Luxemburgo \\
\hline México & 0,954 & 25 & 0,942 & 30 & 0,938 & 32 & Japón, Rep. Corea \\
\hline Países Bajos & 0,961 & 22 & 0,970 & 19 & 0,976 & 17 & Japón \\
\hline Noruega & 0,970 & 14 & 0,976 & 14 & 0,982 & 15 & Japón, Islandia \\
\hline Nueva Zelanda & 0,968 & 16 & 0,974 & 16 & 0,976 & 18 & Japón, Rep. Corea \\
\hline Prom. OCDE & 0,951 & 26 & 0,955 & 24 & 0,960 & 28 & Japón \\
\hline Polonia & 0,934 & 31 & 0,934 & 31 & 0,940 & 31 & Japón, Rep. Corea \\
\hline Portugal & 0,944 & 27 & 0,951 & 25 & 0,966 & 25 & Japón \\
\hline Rep. Eslovaca & 0,918 & 33 & 0,923 & 32 & 0,921 & 34 & Japón, Rep. Corea \\
\hline Eslovenia & 0,935 & 30 & 0,951 & 26 & 0,966 & 26 & Japón, Islandia, Lux \\
\hline Suecia & 0,9856 & 6 & 0,985 & 8 & 0,985 & 13 & Japón, Islandia \\
\hline Turquía & 1 & 1 & 0,916 & 34 & 0,932 & 33 & Turquía, Japón, Rep. Corea, Estonia \\
\hline Estados Unidos & 0,944 & 28 & 0,945 & 28 & 0,948 & 30 & Japón \\
\hline Promedio & 0,960 & & 0,963 & & 0,971 & & \\
\hline
\end{tabular}

Nota: resultados DEA ajustado por quinquenios y rezago del gasto total en salud por BCC-O.

Fuente: elaboración propia. 
Tabla 3A. Modelo DEA para OCDE y ALC

\begin{tabular}{|c|c|c|c|c|c|c|c|c|}
\hline \multicolumn{3}{|c|}{1995} & \multicolumn{3}{|c|}{2005} & \multicolumn{3}{|c|}{2014} \\
\hline \multicolumn{3}{|c|}{ Modelo BCC-O } & \multicolumn{3}{|c|}{ Modelo BCC-O } & \multicolumn{3}{|c|}{ Modelo BCC-O } \\
\hline Rank & DMU (País) & Puntuación & Rank & DMU (País) & Puntuación & Rank & DMU (País) & Puntuación \\
\hline 1 & Japón & 1 & 1 & Islandia & 1 & 1 & República Dominicana & 1 \\
\hline 2 & República de Corea & 1 & 2 & Jamaica & 1 & 2 & Japón & 1 \\
\hline 3 & Suecia & 1 & 3 & Japón & 1 & 3 & Luxemburgo & 1 \\
\hline 4 & Turquía & 1 & 4 & República de Corea & 1 & 4 & España & 0,9999 \\
\hline 5 & Ecuador & 0,9908 & 5 & Luxemburgo & 1 & 5 & República de Corea & 0,9972 \\
\hline 6 & Luxemburgo & 0,9893 & 6 & Suiza & 0,9916 & 6 & Islandia & 0,9957 \\
\hline 7 & Islandia & 0,9863 & 7 & Israel & 0,9894 & 7 & Israel & 0,9949 \\
\hline 8 & Cuba & 0,9861 & 8 & Australia & 0,9868 & 8 & Italia & 0,9942 \\
\hline 9 & Suiza & 0,9859 & 9 & Italia & 0,9861 & 9 & Suiza & 0,9912 \\
\hline 10 & Italia & 0,9828 & 10 & Suecia & 0,9843 & 10 & Australia & 0,988 \\
\hline 11 & España & 0,9804 & 11 & Chile & 0,9817 & 11 & Chile & 0,9871 \\
\hline 12 & Canadá & 0,9804 & 12 & Canadá & 0,9801 & 12 & Francia & 0,9855 \\
\hline 13 & Australia & 0,9785 & 13 & España & 0,9795 & 13 & Jamaica & 0,9844 \\
\hline 14 & Noruega & 0,9785 & 14 & Francia & 0,9785 & 14 & Grecia & 0,983 \\
\hline 15 & Chile & 0,9785 & 15 & Noruega & 0,977 & 15 & Irlanda & 0,9829 \\
\hline 16 & Francia & 0,9776 & 16 & Irlanda & 0,977 & 16 & Noruega & 0,9805 \\
\hline 17 & Grecia & 0,9755 & 17 & Nueva Zelanda & 0,9747 & 17 & Canadá & 0,9805 \\
\hline 18 & Israel & 0,9738 & 18 & Países Bajos & 0,9685 & 18 & Suecia & 0,9805 \\
\hline 19 & Países Bajos & 0,9732 & 19 & Austria & 0,9683 & 19 & Finlandia & 0,9784 \\
\hline 20 & Finlandia & 0,9675 & 20 & Grecia & 0,9672 & 20 & Turquía & 0,9757 \\
\hline 21 & Jamaica & 0,9667 & 21 & República Dominicana & 0,9664 & 21 & Reino Unido & 0,9752 \\
\hline 22 & Bélgica & 0,9661 & 22 & Reino Unido & 0,9649 & 22 & Nueva Zelanda & 0,9739 \\
\hline 23 & Reino Unido & 0,9661 & 23 & Venezuela & 0,9643 & 23 & Austria & 0,9731 \\
\hline 24 & Costa Rica & 0,9653 & 24 & Bélgica & 0,9641 & 24 & Países Bajos & 0,9727 \\
\hline 25 & Nueva Zelanda & 0,9648 & 25 & Alemania & 0,9635 & 25 & Venezuela & 0,9705 \\
\hline 26 & Austria & 0,9639 & 26 & Finlandia & 0,9624 & 26 & Portugal & 0,9693 \\
\hline 27 & Alemania & 0,9608 & 27 & Costa Rica & 0,9595 & 27 & Eslovenia & 0,9681 \\
\hline 28 & Irlanda & 0,9547 & 28 & Promedio OCDE & 0,9551 & 28 & Alemania & 0,9672 \\
\hline 29 & México & 0,9537 & 29 & Perú & 0,9533 & 29 & Perú & 0,965 \\
\hline 30 & Promedio OCDE & 0,9518 & 30 & Portugal & 0,953 & 30 & Bélgica & 0,9641 \\
\hline 31 & Estados Unidos & 0,9508 & 31 & Cuba & 0,9513 & 31 & Dinamarca & 0,9636 \\
\hline 32 & Portugal & 0,9469 & 32 & Dinamarca & 0,9502 & 32 & Polonia & 0,9631 \\
\hline 33 & Dinamarca & 0,9456 & 33 & México & 0,9492 & 33 & Estonia & 0,9618 \\
\hline 34 & Barbados & 0,9437 & 34 & Eslovenia & 0,9474 & 34 & México & 0,9587 \\
\hline 35 & Polonia & 0,9349 & 35 & Estados Unidos & 0,9458 & 35 & Promedio OCDE & 0,9586 \\
\hline 36 & Belice & 0,933 & 36 & República Checa & 0,9444 & 36 & Costa Rica & 0,9543 \\
\hline 37 & Panamá & 0,9319 & 37 & Polonia & 0,9433 & 37 & República Checa & 0,9499 \\
\hline
\end{tabular}

Lecturas de Economía -Lect. Econ. - No. 91. Medellín, julio-diciembre 2019 
Sanmartín-Durango et al.: Eficiencia del gasto en salud en la OCDE y ALC...

Tabla 3A. Continuación

\begin{tabular}{|c|c|c|c|c|c|c|c|c|}
\hline \multicolumn{3}{|c|}{1995} & \multicolumn{3}{|c|}{2005} & \multicolumn{3}{|c|}{2014} \\
\hline \multicolumn{3}{|c|}{ Modelo BCC-O } & \multicolumn{3}{|c|}{ Modelo BCC-O } & \multicolumn{3}{|c|}{ Modelo BCC-O } \\
\hline Rank & DMU (País) & Puntuación & Rank & DMU (País) & Puntuación & Rank & DMU (País) & Puntuación \\
\hline 38 & Eslovenia & 0,9299 & 38 & Barbados & 0,9431 & 38 & Cuba & 0,9498 \\
\hline 39 & Uruguay & 0,9246 & 39 & Estonia & 0,9399 & 39 & Estados Unidos & 0,9444 \\
\hline 40 & República Eslovaca & 0,9219 & 40 & Ecuador & 0,9373 & 40 & Panamá & 0,9387 \\
\hline 41 & Venezuela & 0,9206 & 41 & Panamá & 0,9372 & 41 & Uruguay & 0,9287 \\
\hline 42 & República Checa & 0,9188 & 42 & Bahamas & 0,9298 & 42 & República Eslovaca & 0,9279 \\
\hline 43 & República Dominicana & 0,9102 & 43 & Uruguay & 0,9232 & 43 & Granada & 0,9246 \\
\hline 44 & Santa Lucía & 0,9077 & 44 & Turquía & 0,9220 & 44 & Santa Lucía & 0,9212 \\
\hline 45 & Perú & 0,9064 & 45 & Belice & 0,9217 & 45 & Hungría & 0,9208 \\
\hline 46 & Bahamas & 0,8960 & 46 & República Eslovaca & 0,9177 & 46 & Barbados & 0,9159 \\
\hline 47 & Honduras & ,8959 & 47 & Santa Lucía & 0,9176 & 47 & Suriname & 0,9127 \\
\hline 48 & Trinidad y Tobago & 0,8955 & 48 & Colombia & 0,9144 & 48 & Ecuador & 0,9126 \\
\hline 49 & Promedio ALyC & 0,8831 & 49 & Promedio ALyC & 0,9086 & 49 & Bahamas & 0,9114 \\
\hline 50 & Paraguay & 0,8826 & 50 & Granada & 0,9075 & 50 & Promedio ALyC & 0,9102 \\
\hline 51 & Guatemala & 0,8790 & 51 & Nicaragua & 0,9049 & 51 & Nicaragua & 0,9004 \\
\hline 52 & Hungría & 0,8775 & 52 & Paraguay & 0,8984 & 52 & Guatemala & 0,8998 \\
\hline 53 & Colombia & 0,8729 & 53 & Hungría & 0,8868 & 53 & Colombia & 0,8989 \\
\hline 54 & Granada & 0,8715 & 54 & Trinidad y Tobago & 0,8803 & 54 & Brasil & 0,8987 \\
\hline 55 & Estonia & 0,8560 & 55 & Honduras & 0,8773 & 55 & Belice & 0,8950 \\
\hline 56 & Suriname & 0,8525 & 56 & Brasil & 0,8769 & 56 & Trinidad y Tobago & 0,8940 \\
\hline 57 & Brasil & 0,8525 & 57 & Salvador & 0,8695 & 57 & Salvador & 0,8911 \\
\hline 58 & Salvador & 0,8504 & 58 & Guatemala & 0,8682 & 58 & Honduras & 0,8816 \\
\hline 59 & Nicaragua & 0,8498 & 59 & Suriname & 0,8584 & 59 & Paraguay & 0,8742 \\
\hline 60 & Guyana & 0,8418 & 60 & Guyana & 0,8297 & 60 & Guyana & 0,8685 \\
\hline 61 & Bolivia & 0,7871 & 61 & Bolivia & 0,8059 & 61 & Bolivia & 0,8526 \\
\hline 62 & Haití & 0,7079 & 62 & Haití & 0,7914 & 62 & Haití & 0,7609 \\
\hline
\end{tabular}

Nota: resultados DEA ajustado con ambos grupos BCC-O.

Fuente: elaboración propia.

\section{Referencias}

Alfonso, A. \& St. Aubyn, M. (2004). Non-Parametric Approaches to Education and Health Expenditure Efficiency in OECD Countries (ISEG-UTL Economics Working Paper No. 1/2004/DE/CISEP/UECE. Recuperado de Social Sciense Research Network -SSRN-: http://www.ssrn.com/ abstract $=498383$ 
Alfonso A. (2010). Eficiencia en Salud Pública. Archivo Médico de Camagüey, 14(5). Recuperado de: http://www.redalyc.org/articulo.oa?id $=211116131020$

Banco Mundial (2015). Datos. Recuperado de: https://datos.banco mundial.org/

Banker, R., Charnes, A. \& Cooper, W. W. (1984). Some Models for Estimating Technical and Scale Inefficiencies in Data Envelopment Analysis. Management science, 30(9), 1078-1092.

Bosch Roca, N., Chaparro P. \& Suárez Pandiello, J. (diciembre, 1995). "Medición de la eficiencia en la provisión de servicios públicos locales". En V Congreso Nacional de Economía: Economía de Los Servicios, Las Palmas de Gran Canaria).

Charnes, A.; Cooper, W. W., Rhodes, E. (1978). A Data Envelopment Analysis Approach to Evaluation of the Program Follow through Experiment in US Public School Education. Estados Unidos: Division of Research, Graduate School of Business Administration, Hardvard University.

Clements, B., Coady, D. \& Gupta, S. (2012). The Economics of Public Health Care Reform in Advanced and Emerging Economies. Washington: International Monetary Fund Publications.

Cook, W. D., Tone, K. \& Zhu, J. (2014). Data Envelopment Analysis: Prior to Choosing a Model. Omega, 44, 1-4.

Dieleman, J. L., Templin, T., Sadat, N., Reidy, P., Chapin, A., Foreman, K., Haakenstad, A. et al. (2016). National Spending on Health by Source for 184 Countries between 2013 and 2040. The Lancet, 387(10037), 2521-2535. Recuperado de: http://linkinghub.elsevier.com/retrieve/pii/ S0140673616301672

Evans, D. B., Tandon, A., Murray, C. J. L. \& Lauer, J. A. (2000). The Comparative Efficiency of National of Health Systems in Producing Health: An Analysis of 191 Countries (GPE Discussion Paper Series, No. 29). Recuperado del sitio web de la Organización Mundial de la Salud: www.who.int/healthinfo/paper29.pdf 
Sanmartín-Durango et al.: Eficiencia del gasto en salud en la OCDE y ALC...

Farrell, M. J. (1957). The measurement of productive efficiency. Journal of the Royal Statistical Society: Series A (General), 120(3), 253-281.

Grossman, M. (1999). The Human Capital Model of the Demand for Health (NBER Working Paper, No. 7078). Recuperado del sitio web de The National Bureau of Economic Research. http://www.nber.org/ papers/w7078

Heller, P. S. \& Hauner, D. (2006). Fiscal Policy in the Face of Long-Term Expenditure Uncertainties. International Tax and Public Finance, 13(4), 325-50.

Huicho, L. Segura, E., Huayanay-Espinoza, C., Niño de Guzman, J., Restrepo-Méndez, M. C., Tam, Y. et al. (2016). Child Health and Nutrition in Peru within an Antipoverty Political Agenda: A Countdown to 2015 Country Case Study. The Lancet Global Health, 4(6), e414-26.

Jackson, B. \& Ugalde, A. (Eds.) (1987). The Impact of Development and Modern Technologies in Third World Health. Studies in Third World Societies, 34.

Ji, Y-B. \& Lee, C. (2010). Data Envelopment Analysis. The Stata Journal, 10(2), 267-280.

Xu, K., Saksena, P. \& Holly, A. (2011). The Determinants of Health Expenditure: A Country-Level Panel Data Analysis (Working Paper/December 2011). Recuperado del sitio web de la Organización Mundial de la Salud: http://www.who.int/health_financing/documents/report_en_11_deterhe.pdf

Lago, F. P., Geri, M., Moscoso, N. S. \& Monterubbianes, P. D. (2013). Gasto total en salud resultados, Ciencias Económicas, 31(2), 101-116.

Lam Díaz, R. M. \& Hernández Ramírez, P. (2008). Los términos: eficiencia, eficacia y efectividad ¿son sinónimos en el área de la salud?. Revista Cubana de Hematologia, Inmunologia y Hemoterapia, 24(2), 1-6. 
Machado, R. (2006). ¿Gastar más o gastar mejor?: la eficiencia del gasto público en América Central y República Dominicana. Recuperado de: https://publications.iadb.org/es/gastar-mas-o-gastar-mejor-laeficiencia-del-gasto-publico-en-america-central-y-republica-dominicana

Moreno-Serra, R. \& Smith, P. C. (2015). Broader health coverage is good for the nation's health: evidence from country level panel data. Journal of the Royal Statistical Society. Series A: Statistics in Society, 178(1), 101-124.

Muñoz-Repiso, J. M. C. (2001). La eficiencia del sector público: métodos de evaluación y organismos responsables: el caso de España. Espańa: Instituto de Estudios Fiscales

Organización Mundial de la Salud (OMS) (2010). Informe sobre la salud en el mundo: la financiación de los sistemas de salud: el camino hacia la cobertura universal: resumen (No. WHO/IER/WHR/10.1). Ginebra: Autor.

Organización Mundial de la Salud (OMS) \& Organización Panamericana de la Salud (OPS) (2014). Estrategia para el acceso universal a la salud y la cobertura universal de salud. Recuperado de: https://www.paho.org/uhexchange/index.php/en/uhexchange-docu ments/informacion-tecnica/27-estrategia-para-el-acceso-universal-a-lasalud-y-la-cobertura-universal-de-salud/file

Payne, G., Laporte, A., Deber, R. \& Coyte, P. C. (2007). Counting Backward to Health Care's Future: Using Time-to-Death Modeling to Identify Changes in End-of-Life Morbidity and the Impact of Aging on Health Care Expenditures. The Milbank Quarterly, 85(2), 213-257. doi: 10.1111/j.1468-0009.2007.00485.x

Reidpath, D. D. \& Allotey, P. (2003). Infant mortality rate as an indicator of population health. Journal of Epidemiology and Community Health, 57(5), 344-346.

Rivera, B. (2001). The effects of public health spending on self-assessed health status: an ordered probit model. Applied Economics, 33(10), 1313-1319. doi: 10.1080/00036840010007146 
Sanmartín-Durango et al.: Eficiencia del gasto en salud en la OCDE y ALC...

Schwartzmann, L. (2003). Calidad de vida relacionada con la salud: aspectos conceptuales. Ciencia y enfermería, 9(2), 9-21.

Xu, K., Saksena, P., Jowett, M., Indikadahena, C., Kutzin, J. \& Evans, D. (2010). Exploring the thresholds of health expenditure for protection against financial risk. World Health Report Background Paper, No. 19. World Health Organization. 